\title{
REDUCED HAUSDORFF DIMENSION AND CONCENTRATION-CANCELLATION FOR TWO-DIMENSIONAL INCOMPRESSIBLE FLOW
}

RONALD J. DIPERNA AND ANDREW MAJDA

\section{CONTENTS}

1. Introduction

2. Defect measures

3. Concentration-cancellation

4. Uniform distribution functions

5. Chebyshev inequalities

6. $L^{p}$ velocity estimates

7. Space-time defects

Appendix: Truncation, interpolation, and Hölder continuity in time

\section{INTRODUCTION}

The Euler equations for an inviscid incompressible 2-D fluid flow are given by

$$
\begin{aligned}
& D v / D t=-\nabla p, \quad x \in R^{2}, t>0, \\
& \operatorname{div} v=0, \quad v(x, 0)=v_{0}(x)
\end{aligned}
$$

where $v={ }^{t}\left(v_{1}, v_{2}\right)$ is the fluid velocity, $p$ is the scalar pressure, $D v / D t=$ $\partial v / \partial t+(v \cdot \nabla) v$, and $v_{0}$ is an initial incompressible velocity field, i.e. $\operatorname{div} v_{0}=0$

In this paper, we study the detailed limiting behavior of approximate solution sequences for 2-D Euler with vortex sheet initial data. A sequence of smooth velocity fields $v^{\varepsilon}(x, t)$ is an approximate solution sequence for $2-\mathrm{D}$ Euler provided that $v$ is incompressible, i.e. $\operatorname{div} v=0$, and satisfies the following properties:

(1) The velocity fields $v^{\varepsilon}$ have uniformly bounded local kinetic energy, i.e.

$$
\max _{0 \leq t \leq T} \int_{|x| \leq R}\left|v^{\varepsilon}(x, t)\right|^{2} d x \leq C
$$

for any $R, T>0$.

Received by the editors November 3, 1986.

1980 Mathematics Subject Classification (1985 Revision). Primary 35D99, 76C99.

First author partially supported by National Science Foundation grant \#83-01135.

Second author partially supported by National Science Foundation grant \#86-11110. 
(2) The corresponding vorticity, $\omega^{\varepsilon}=\operatorname{curl} v^{\varepsilon}$, is uniformly bounded in $L^{1}$, i.e.

$$
\max _{0 \leq t \leq T} \int\left|\omega^{\varepsilon}(x, t)\right| d x \leq C
$$

for any $T>0$.

(3) The velocity field $v^{\varepsilon}$ is weakly consistent with 2-D Euler, i.e. for all smooth test functions, $\varphi \in C^{\infty}\left(R^{2} \times(0, \infty)\right)$ with $\operatorname{div} \varphi=0$,

$$
\lim _{\varepsilon \rightarrow 0} \iint \varphi_{t} \cdot v^{\varepsilon}+\nabla \varphi: v^{\varepsilon} \otimes v^{\varepsilon} d x d t=0 .
$$

Here $v \otimes v=\left(v_{i} v_{j}\right), \nabla \varphi=\left(\partial \varphi_{i} / \partial x_{j}\right)$, and $A: B$ denotes the matrix product $\sum_{i, j} a_{i j} b_{i j}$. We remark in passing that the definition of approximate solution sequence, as given in $\S 1$ of [4], includes the requirements that $v^{\varepsilon}$ vanishes uniformly as $|x| \rightarrow \infty$ and also that $v^{\varepsilon}$ is uniformly Lipschitz in some negative Sobolev space in order to interpret the initial data in a weak sense in the limit. These precise definitions are not needed here except for the discussion in the appendix which links the results here with those presented in [3, 4].

Approximate solution sequences for 2-D Euler can be generated in a variety of ways. Three important processes for generating approximate 2-D Euler sequences with a fixed initial velocity field $v_{0}$ are the following: smoothing the initial data, the zero diffusion limit of 2-D Navier-Stokes solutions, and the algorithm of computational vortex dynamics. Each of these is described in detail in the companion paper of the authors [4]. The requirements of a uniform local kinetic energy bound and weak consistency with 2-D Euler stated in (1.2) and (1.4) are natural conditions on approximate solution sequences. The uniform $L^{1}$-bound on the vorticity (1.3) enables us to treat approximate solution sequences with initial data that regularize a fixed initial incompressible velocity field $v_{0}$ with the following two properties:

The velocity $v_{0}$ has locally finite energy and the vorticity $\omega_{0}$ is a Radon measure with compact support.

Initial data with these properties are important from the applied point of view since they arise in the study of the evolution of vortex sheets (see [3, 4] for additional discussion and references). We call velocity fields satisfying (1.5) vortex sheet data. Indeed, since vorticity is transported in 2-D flows, i.e. $D \omega / D t=0$, the uniform $L^{1}$ control of vorticity for approximate velocity fields postulated in (1.3) is a natural condition to impose on approximate solution sequences with vortex sheet initial data.

The weak formulation of the 2-D Euler equations is obtained by multiplying (1.1) by suitable test functions and integrating by parts. A function $v \in L_{\text {loc }}^{2}\left(R^{2} \times(0, \infty)\right)$ is a weak solution of 2-D Euler provided that

$$
\iint\left(\varphi_{t} \cdot v+\nabla \varphi: v \otimes v\right) d x d t=0
$$


for all test functions $\varphi \in C_{0}^{\infty}\left(R^{2} \times(0, \infty)\right)$ with $\operatorname{div} \varphi=0$ and $v$ is incompressible in the weak sense, i.e.

$$
\iint \nabla \varphi \cdot v d x d t=0
$$

for all scalar test functions $\varphi \in C_{0}^{\infty}$.

This paper as well as the authors' companion paper [4] are concerned with the following basic questions:

If $v_{0}$ is vortex sheet initial data, is there a weak solution of 2-D Euler on $R^{2} \times(0, \infty)$ with initial data $v_{0}$ ? If $v^{\varepsilon}$ is an (1.7) approximate solution sequence for 2-D Euler, does $v^{\varepsilon}$ converge to a weak solution of 2-D Euler as $\varepsilon \downarrow 0$ ? Do new phenomena occur in the limiting process?

To the authors' knowledge, this paper as well as the companion papers [3, 4] are the first to address these questions in the mathematical literature. There are some special results for piecewise analytic initial data utilizing CauchyKowaleski theorems which are referenced in [4].

For a general approximate solution sequence satisfying (1.2)-(1.4), it is not difficult to prove that there is a velocity field $v \in L_{\text {loc }}^{2}\left\{R^{2} \times(0, \infty)\right\}$ so that a subsequence $v^{\varepsilon}$ satisfies

$$
\begin{gathered}
\left\|v^{\varepsilon}-v\right\|_{L^{p}(\Omega)} \rightarrow 0, \quad 1 \leq p<2, \\
v^{\varepsilon} \rightarrow v \quad \text { weakly in } L^{2}(\Omega),
\end{gathered}
$$

as $\varepsilon \rightarrow 0$. Here and below, $\Omega$ is any space-time region of the form $\Omega=$ $\{(x, t)|| x \mid \leq R, 0<t<T\}$ and $L^{p}$ is the standard Lebesgue space. The fact (1.8) is proved in Theorem 1.1 of [4]. In [3, 4] several explicit examples of exact smooth solution sequences for 2-D Euler are constructed satisfying the uniform bounds in (1.2)-(1.4), but so that new phenomena occur in the limiting process, a finite amount of kinetic energy concentrates on a set of measure zero and

$$
\int_{\Omega}|v|^{2}<\liminf \int_{\Omega}\left|v^{\varepsilon}\right|^{2}
$$

As a consequence of (1.9), the issue of whether $v$ defines a weak solution of 2-D Euler is subtle since

$$
\lim _{\varepsilon \rightarrow 0} \int_{\Omega} \varphi v_{i}^{\varepsilon} v_{j}^{\varepsilon} \neq \int_{\Omega} \varphi v_{i} v_{j}
$$

for suitable test functions $\varphi \in C_{0}^{\infty}(\Omega)$ and the definition of weak solution from (1.6) involves combinations of such quadratic terms.

In this paper we introduce a new tool to measure weak convergence, the reduced defect measure. Our main results are the following. We prove that the reduced defect measure associated with an approximate sequence of velocity fields for 2-D Euler always concentrates on a space-time set of Hausdorff dimension less than or equal to one. This concentration phenomena can occur 
despite the fact that the standard weak-star defect measure introduced by Lions $[6,7]$ may attach positive mass to space-time sets of positive three-dimensional Lebesgue measure. Examples from $[3,4]$ prove that this result is sharp. Furthermore, under the assumption that the reduced defect measure concentrates on a space-time set with Hausdorff dimension strictly less than one, we prove that, despite the phenomena in (1.9) and (1.10), there is concentration-cancellation and $v$ is a weak solution of the 2-D Euler equations.

The remainder of the introduction includes a precise description of these results as well as a sketch of the overall argument which links together several technical results presented in detail in subsequent sections. The results presented here are part of a general program of the authors to study the development of concentrations and oscillations in fluid flows in both two and three space dimensions. The interested reader can consult $[3,4]$ for a discussion of severat additional phenomena which occur in 3-D fluid flows as well as a detailed description of concentrations in 2-D flows from another viewpoint. In this connection we mention that if we replace the bound for vorticity in (1.3) by the slightly stronger uniform $L^{p}$ bound for some $p$ with $1<p$, then no concentrations as described in (1.9) occur and $v^{\varepsilon}$ converges strongly to $v$ in $L^{2}(\Omega)$ with $v$ a classical weak solution for 2-D Euler (see [4]).

One of our main tools in assessing the size of energy concentrations is the reduced defect measure $\theta$ associated with an arbitrary $L^{2}$ weakly convergent sequence $\left(v^{\varepsilon} \rightarrow v\right)$ through the definition,

$$
\theta(E)=\limsup _{\varepsilon \rightarrow 0} \iint_{E}\left|v^{\varepsilon}-v\right|^{2} d x d t
$$

where $E$ is a Borel subset of $R^{2} \times R^{+}$. The set function $\theta$ is a nonnegative outer measure which vanishes precisely on those sets $E$ where $v^{\varepsilon}$ converges strongly to $v ; \theta$ is concentrated on the $L^{2}$ exceptional sets of the sequence $v_{\varepsilon}$ where convergence is weak but not strong.

Definition. A finitely subadditive outer measure $\theta$ is concentrated inside a set $E$, i.e. $E$ is a concentration set for $\theta$, if $E^{c}$ is a countable union of null sets of $\theta$.

Notice that in general the reduced defect measure $\theta$ is a finitely subadditive outer measure. Typically, it is not continuous from below. In particular, the countable union of null sets need not be a null set, as is the case for a countably additive outer measure.

The weak star defect measure introduced by Lions in the theory of concentration compactness $[6,7]$ is the Radon measure $\sigma$ so that

$$
\left|v^{\varepsilon}-v\right|^{2} \rightarrow \sigma \quad \text { weak star. }
$$


The reduced defect measure has two properties which are crucial for the developments here:

For any closed set $F, \theta(F) \leq \sigma(F)$, and the sequence $v^{\varepsilon}$ converges strongly to $v$ on a set $E$ if and only if $\theta(E)=0$.

In particular, at the end of $\S 1$ from [4], several examples are given where $\sigma$ attaches positive weight to a closed set with positive Lebesgue measure for steady sequences satisfying (1.2) and (1.3) while the analysis here guarantees that $\theta$ concentrates on a set with one-dimensional Hausdorff measure. General properties of the reduced defect measure are discussed in $\S 2$.

One of four main results in this paper is an estimate for the Hausdorff dimension of the concentration set for the reduced defect measure. In order to state this theorem, we recall that the weight which $\gamma$-order Hausdorff measure assigns to a set is expressed as a limit of premeasures,

$$
H^{\gamma}(E)=\lim _{r \rightarrow 0} H_{r}^{\gamma}(E)=\sup _{r>0} H_{r}^{\gamma}(E)
$$

with the premeasure $H_{r}^{\gamma}$ given by

$$
H_{r}^{\gamma}(E)=\inf \left\{\sum r_{j}^{\gamma} \mid E \subseteq U B_{j}, r_{j} \leq r\right\} .
$$

Here $\left\{B_{j}\right\}$ is a countable cover of $E$ by open balls. The main part of this paper is a proof of the following theorem.

Theorem 1. Consider any approximate solution sequence for 2-D Euler satisfying (1.2) - (1.4). Then the reduced defect measure $\theta$ of a subsequence is concentrated inside a space-time set $E$ of Hausdorff dimension less than or equal to one, i.e.

$$
H^{1+\delta}(E)=0 \text { for all } \delta>0 .
$$

The examples of fluid flows in $[3,4]$ show that this theorem is sharp. The other main result in this paper connects the Hausdorff dimension of the reduced defect measure and the phenomena of concentration-cancellation mentioned earlier.

Theorem 2. Suppose $v_{\varepsilon}$ is a sequence of 2-D Euler solutions which converges weakly in $L^{2}$ to a vector field $v$. If the reduced defect measure is concentrated inside a space-time set with Hausdorff dimension less than one then $v$ is a weak solution of 2-D Euler, i.e. satisfies (1.6).

The proof of Theorem 2 is given in $\S 3$. This result is a corollary of a stronger result which applies to steady elliptic sequences satisfying (1.2)-(1.4) which we present first in $\S 3$.

The proofs in $\S 3$ reveal a surprisingly robust property of the inertial terms in the equations of incompressible flow. These terms have the form

$$
T(\varphi, v)=\iint \nabla \varphi: v \otimes v \quad \text { with } \operatorname{div} \varphi=0 .
$$


The inertial terms are completely insensitive to concentrations in the energy field so that despite the appearance of concentrations as described in (1.9) and (1.10), the limit is still a weak solution. Our results in $\S 3$ combined with the steady state analysis in $\S \S 4-6$ prove that this is always the case for steady fluid flows with external forces. We need the slightly stronger postulate on the Hausdorff dimension of the concentration set in Theorem 2 for the general time-dependent case. It is not clear that Theorem 2 is valid for general time-dependent approximate solution sequences for 2-D Euler satisfying (1.2)-(1.4). It is an interesting problem to investigate this issue.

The proof of Theorem 2 relies on the rotational invariance of the inertial terms and the following device. For a given test function $\varphi$, we construct a shadow sequence of test functions $\varphi^{\varepsilon}$ which shields $\varphi$ from the kinetic energy defects in $v^{\varepsilon}$ so that $\varphi^{\varepsilon} \rightarrow \varphi$ and

$$
\lim _{\varepsilon \rightarrow 0} T\left(\varphi^{\varepsilon}, v^{\varepsilon}\right)=T(\varphi, v) .
$$

The shadow sequence $\varphi^{\varepsilon}$ depends on the sequence $v^{\varepsilon}$.

The results in $\S \S 4-7$ combine to give the proof of Theorem 1. Actually, the results in $\S \S 4-6$ apply to elliptic sequences which satisfy the uniform kinetic energy and vorticity bounds in (1.2) and (1.3) and thus apply to approximate solution sequences for 2-D Euler at fixed time. In $\S 7$, we piece together the results at fixed time together with various temporal Hölder estimates proved in the Appendix through interpolation inequalities to conclude the proof of Theorem 1.

At any fixed time $t$, the velocity field for a 2-D incompressible flow which vanishes as $|x| \rightarrow \infty$ can be recovered from the vorticity $\omega=\operatorname{curl} v$ through a stream function $\psi$ satisfying

$$
\begin{aligned}
& \Delta \psi=\omega, \\
& v=\nabla^{\perp} \psi={ }^{t}\left(-\psi_{x_{2}}, \psi_{x_{1}}\right) .
\end{aligned}
$$

The stream function $\psi$ is determined within a constant by convolution of $\omega$ with the logarithmic potential. More precisely, the incompressible velocity field is determined directly from the vorticity by the convolution

$$
v(x)=\int_{R^{2}} K(x-y) \omega(y) d y
$$

with the kernel $K$ given by

$$
K(x)=(2 \pi|x|)^{-2}\left(\begin{array}{c}
-x_{2} \\
x_{1}
\end{array}\right) .
$$

In $\S \S 4-6$, we estimate the Hausdorff dimension of elliptic sequences satisfying the following uniform bounds

$$
\int_{|x| \leq R}\left|v^{\varepsilon}\right|^{2}+\int_{|x| \leq R}\left|w^{\varepsilon}\right| d x \leq C(R) .
$$


Through a straightforward truncation argument for approximate solution sequences of 2-D Euler sketched in the Appendix, we assume here and below that the velocity fields $v^{\varepsilon}$ have compact support inside the ball $|x| \leq R$. In this situation there is a steady velocity field $v$ satisfying (1.8) with $\Omega=\{x|| x \mid \leq R\}$. Furthermore, the argument presented in $\S \S 4-6$ yields the following theorem.

Theorem 3. If $v^{\varepsilon}$ converges weakly to $v$ under the uniform energy and vorticity bounds in (1.15), then the reduced defect measure $\theta$ concentrates inside a set I with Hausdorff dimension zero, i.e.

$$
H^{\delta}(I)=0 \text { for all } \delta>0 .
$$

In this argument, we produce a family of closed sets $F_{r} \subseteq \Omega, r>0$, so that

$$
\theta\left(F_{r}\right)=\limsup _{\varepsilon \rightarrow 0} \int_{F_{r}}\left|v^{\varepsilon}-v\right|^{2} d x=0
$$

and the Hausdorff premeasure of the complement satisfies

$$
H_{r}^{\delta}\left(F_{r}^{c}\right) \leq C
$$

where $C$ is a constant independent of $\delta$ and $r$. By first letting $r$ then $\delta$ tend to zero along sequences we deduce Theorem 3 .

The closed sets $F_{r}$ are determined by the uniformization theorem proved in $\S 4$. We introduce the vorticity maximal function

$$
\omega^{\varepsilon}(s, x)=\left|\omega^{\varepsilon}\right|\{B(s, x)\}=\int_{|y-x|<s}\left|\omega^{\varepsilon}\right| d y .
$$

The content of the uniformization theorem is that for a subsequence it is possible to find a closed set $F_{r}$ with complement satisfying (1.17) so that

$$
\sup _{\substack{x \in F_{r} \\ s \leq 1}} s^{-\delta} \omega^{\varepsilon}(s, x) \leq K(r) .
$$

Using the above uniform estimate on the vorticity maximal function on $F_{r}$ we prove in $\S 6$ that this leads to an improved uniform velocity estimate on $F_{r}$ of the form

$$
\int_{F_{r}}\left|v^{\varepsilon}\right|^{q} d x \leq K(r)
$$

for some $q>2$. With the above uniform bound in $L^{q}\left(F_{r}\right)$ for $q>2$ and the fact from (1.8) that $v^{\varepsilon} \rightarrow v$ strongly in $L^{p}(\Omega)$ for any $p<2$, simple interpolation yields the conclusion in (1.16).

The uniformization theorem in $\S 4$ is proved through the use of Riesz potentials and a generalized Chebyschev inequality involving Hausdorff premeasure presented in $\S 5$. This inequality is suggested by earlier work of Federer and Ziemer [5] using capacities instead of Hausdorff premeasures. Actually, Theorem 3 is also valid for appropriate logarithmic Hausdorff measures. Although we do not develop all the necessary details here, we do present several important facets of this generalization here (also see $\S 3$ of [4]). In $\S 7$ we piece together 
space-time exceptional sets by utilizing the arguments just presented at fixed time together with various temporal Hölder estimates which are described in the Appendix.

\section{DEFECT MEASURES}

This section deals with two set-functions which record the loss of compactness in weakly convergent sequences, the weak-star defect measure, and the reduced defect measure. In the context of 2-D incompressible fluid flow and vortex sheet initial data, the weak-star defect measure has already been discussed in $\S 1$ of [4]. The reduced defect measure is the main concept utilized in this paper. The main theoretical advantage of the reduced defect measure is that this measure vanishes on a closed set if and only if there is strong convergence on this set. On the other hand, the weak-star defect measure can attach positive weight to a closed set where there is strong convergence. In fact, the examples of elliptic sequences given at the end of $\S 1$ of [5] provide explicit examples of this phenomena relevant for the 2-D Euler equations with vortex sheet initial data.

The general setting is the following. We consider an arbitrary sequence of functions $u_{\varepsilon}$ so that $u_{\varepsilon}$ converges weakly to $u$, i.e.

$$
u_{\varepsilon} \rightarrow u \text { in } L^{q}, q>1 \text {. }
$$

For simplicity in exposition, we assume that all distributions under discussion have support inside a fixed compact subset of $R^{N}$. Thus, the $L^{q}$ norm of $u_{\varepsilon}$ is uniformly bounded with respect to $\varepsilon$ and the local average of $u_{\varepsilon}$ converges to the local average of $u$,

$$
\lim \int_{E} u_{\varepsilon} d x=\int_{E} u d x
$$

for all measurable sets $E$ in $R^{N}$. We consider the sequence of measures $\sigma_{\varepsilon}$ associated with the densities $\left|u_{\varepsilon}-u\right|^{q}$ :

$$
\sigma_{\varepsilon}(E)=\int_{E}\left|u_{\varepsilon}-u\right|^{q} d x .
$$

Since the total mass of $\sigma_{\varepsilon}$ is uniformly bounded, there exists a subsequence that converges in the weak-star topology to a measure $\sigma$, i.e.

$$
\lim \int \varphi d \sigma_{\varepsilon}=\int \varphi d \sigma
$$

for all continuous functions $\varphi$. The limiting measure $\sigma$ encodes information on the sets where convergence is weak but not strong.

Definition 2.1. Assume that $u_{\varepsilon}$ converges in the weak topology of $L^{q}$ to $u$ and that $\left|u_{\varepsilon}-u\right|^{q}$ converges in the weak-star topology. The measure

$$
\sigma=w^{*}-\lim \left|u_{\varepsilon}-u\right|^{q}
$$

is called the weak-star defect measure of the sequence $u_{\varepsilon}$. 
In the special case of noncompact Sobolev embeddings, the weak-star defect measure was introduced by P. L. Lions (see [6, 7] as well as the discussion in $\S 1$ of [5] for the relation between the Lions theory and problems with vortex sheet initial data).

One general problem is to determine the relationship between the vanishing of $\sigma$ and strong convergence. If $u_{\varepsilon}$ converges strongly in $L^{q}$ to $u$ on a set $E$, does $\sigma(E)=0$ ? If $\sigma$ vanishes on $E$, does $u_{\varepsilon}$ converge strongly on $E$ ? In the special case where $E=R^{N}$ it is clear that $u_{\varepsilon}$ converges strongly to $u$ if and only if $\sigma$ vanishes identically. In general the structure of $E$ influences the answers and indicates various features of sensitivity which motivate our introduction of the reduced defect measure. The answer to the above questions when the set $E$ is either closed or open is contained in the following.

Lemma 2.1. (1) If $\sigma$ vanishes on a closed set $F$, then the restriction of $u_{\varepsilon}$ to $F$ converges strongly to the restriction of $u$ to $F$.

(2) If $u_{\varepsilon}$ converges strongly to $u$ on an open set $G$ then $\sigma(G)=0$.

In general, strong convergence on a set $E$ is not equivalent to the vanishing of $\sigma$ on $E$. The above lemma is an immediate consequence of the following characterization of weak-star convergence for measures.

Proposition. Suppose $\sigma_{\varepsilon}$ is an arbitrary sequence of measures. Then $\sigma=w^{*}-$ $\lim \sigma_{\varepsilon}$ if and only if the following two conditions hold:

$$
\sigma(G) \leq \liminf \sigma_{\varepsilon}(G)
$$

for all open sets $G$ and

$$
\sigma(F) \geq \lim \sup \sigma_{\varepsilon}(F)
$$

for all closed sets $F$.

A sketch of the key part of this proposition is given at the end of this section. To summarize the above proposition, the evaluation functional on measures $L_{E}(\sigma)=\sigma(E)$ is lower semicontinuous if $E$ is open and upper semicontinuous if $E$ is closed in the weak-star topology. In general, there is no preferred inequality if $E$ is neither open nor closed and the operations of restriction and weak-star limit do not commute.

The second inequality in the above proposition motivates the definition of the reduced defect measure which we give below and have already mentioned in the Introduction. Indeed, the examples given at the end of $\S 1$ from [5] are examples of elliptic sequences satisfying the additional structure for vortex sheets for 2-D incompressible flow where the weak star-defect measure $\sigma$ attaches positive weight to closed sets where the convergence is strong; the strong convergence claimed above follows from the analysis given in this paper. Thus, the inequalities in the proposition are often strict inequalities.

The facts above motivate the following definition. 
Definition 2.2. Assume that $u_{\varepsilon}$ converges weakly to $u$ in $L^{q}\left(R^{n}\right), q>1$. The outer measure $\theta$ defined by

$$
\theta(E)=\limsup \int_{E}\left|u_{\varepsilon}-u\right|^{q} d x
$$

is called the reduced defect measure of the sequence $u_{\varepsilon}$.

Remark. The following important fact follows from the definition.

$\theta$ vanishes on $E$ if and only if $u_{\varepsilon}$ converges strongly to $u$ in $L^{q}(E)$.

Furthermore, from the second inequality in the proposition above, we see that for any closed set $F$,

$$
\theta(F)=\lim \sup \int\left|u_{\varepsilon}-u\right|^{q} d x \leq \sigma(F) .
$$

This inequality implies that $\theta$ might be small on closed sets where the measure $\sigma$ is large. In fact, in this paper we prove that $\theta(F)$ is extremely small on closed sets $F$ where $\sigma$ attaches positive weight.

Remark. Simple examples show that in general $\theta$ is a finitely subadditive outer measure.

Finally, we sketch the proof of the first inequality in the proposition. Thus we assume that $\sigma=w^{*}-\lim \sigma_{\varepsilon}$ and deduce that $\sigma(G) \geq \liminf \sigma_{\varepsilon}(G)$ for all open sets $G$. Since $\sigma$ is a Radon measure, $\sigma$ is inner (outer) regular. Thus for any $\delta>0$, there exists a compact $K$ with $K \subset G$ and

$$
\sigma(G)-\delta \leq \sigma(K) \text {. }
$$

By Urysohn's lemma, there exists $f \in C_{0}\left(R^{N}\right)$ so that $f$ satisfies $0 \leq f \leq 1$ and also

$$
\begin{array}{ll}
f(x)=1, & x \in K, \\
f(x)=0, & x \in G^{c} .
\end{array}
$$

Therefore,

$$
\sigma(G)-\delta \leq \sigma(K) \leq \int f d \sigma=\lim \int_{G} f d \sigma_{\varepsilon} \leq \underline{\lim } \int f d \sigma_{\varepsilon} \leq \underline{\lim } \sigma_{\varepsilon}(G) .
$$

We prove the second inequality in the proposition by using the outer regularity of $\sigma$ in a similar fashion. The proof of the fact that the two inequalities guarantees weak convergence utilizes approximation by simple functions and is left as an exercise for the reader.

\section{Concentration-CANCEllation}

In this section we consider exact solution sequences satisfying the basic bounds (1.2)-(1.4). For arbitrary vortex sheet initial data such sequences are generated by smoothing the initial data (see $\S 1$ of [5]). Here we prove Theorem 2 as described in the Introduction. First, we formulate and prove a stronger 
result for steady solution sequences for 2-D Euler with external forces and satisfying uniform local energy and $L^{1}$ vorticity bounds. This steady state theorem provides the first link between the Hausdorff dimension of the concentration set of the reduced defect measure and concentration-cancellation. The proof of Theorem 2 is a simple corollary of the proof of this steady state theorem.

The formulation of the steady problem is the following. Consider a sequence of fields $v_{\varepsilon}=v_{\varepsilon}(x)$ on $R^{2}$ such that

$$
\operatorname{div} v_{\varepsilon} \otimes v_{\varepsilon}=f_{\varepsilon}
$$

where $f_{\varepsilon}$ converges weakly to $f$ in $L^{1}$. Assume that $v_{\varepsilon}$ converges weakly in $L^{2}$ to $v$ and that the corresponding reduced defect measure $\theta$ is concentrated on a set with Hausdorff dimension less than one. As indicated earlier this hypothesis holds if the total vorticity is uniformly bounded in the sense that

$$
\text { total mass } \omega_{\varepsilon} \leq c
$$

where $\omega_{\varepsilon}$ is viewed as a measure. Indeed, as described in Theorem 3 of the Introduction, the uniform bound (3.2) guarantees that $\theta$ is concentrated on a set with zero Hausdorff dimension. However, for the purpose of establishing the weak closure property it suffices to assume that $v_{\varepsilon}$ converges strongly on the complement of a set with dimension less than one.

As described in the Introduction, the precise interpretation of the latter statement is the following. There exists a parameter $\gamma<1$ and a family of sets $F_{r}, r>0$, such that

$$
H_{r}^{\gamma}\left(F_{r}^{c}\right) \leq c
$$

where $c$ is independent of $r$ and

$$
\lim \int_{F_{r}}\left|v_{\varepsilon}-v\right|^{2} d x=0 .
$$

We may assume without loss of generality that $F_{r}^{c}$ is a countable union of open balls $B_{j}$ with $\gamma$-summable radii $r_{j}$ :

$$
\sum r_{j}^{\gamma} \leq c ; \quad r_{j} \leq r .
$$

In short, only fine open covers enter the analysis.

Theorem 3.1. Suppose $v$ is the weak $L^{2}$ limit of a steady 2-D Euler sequence $v_{\varepsilon}$ for which the reduced defect measure is concentrated inside a set with Hausdorff dimension less than one. Then $v$ solves

$$
\operatorname{div} v \otimes v=f
$$

if $f_{\varepsilon}$ converges weakly to $f$ in $L^{1}$.

Proof. The essential ingredients are the covariant structure of the equations and the fact that $v_{\varepsilon}$ converges strongly to $v$ on the complement of a set whose 
orthogonal projection onto any line $L$ has arbitrarily small one-dimensional Lebesgue measure.

The appropriate family of sets is provided by $F_{r}$. If $P_{L}$ denotes orthogonal projection onto $L$ and if $m$ denotes one-dimension Lebesgue measure on $L$ then

$$
m\left\{P_{L}\left(F_{r}^{c}\right)\right\} \leq c \sum r_{j}=\sum r_{j}^{\gamma} r_{j}^{1-\gamma} \leq c r^{1-\gamma} .
$$

Since $\gamma<1$ and $r$ may be taken arbitrarily small, the Lebesgue measure of the projection, i.e. the sum of the lengths of the intervals on $L$ corresponding to the balls $B_{j}$, is arbitrarily small.

The goal is to prove that

$$
\int \nabla \varphi: v \otimes v d x=-\int(\varphi, f) d x
$$

for all $\varphi$ in the space of smooth divergence-free vector fields with compact support. The tensor contraction takes the following form in components:

$$
\nabla \varphi: v \otimes v=\sum\left(\partial \varphi_{i} / \partial x_{j}\right) v_{i} v_{j}
$$

where $v_{i}$ denotes the components of the vector $v$ in a specified orthonormal frame. The choice of the frame is arbitrary.

Since the problem is local we may assume without loss of generality that all of the members of the sequence $v_{\varepsilon}$ are supported inside a fixed compact set. Indeed, for this reason it suffices to assume that the restriction of $\theta$ to any compact set has Hausdorff dimension less than one.

For technical reasons it is convenient to introduce a scalar potential function $\eta$ whose orthogonal gradient corresponds to $\varphi$ :

$$
\varphi=\left(\varphi_{1}, \varphi_{2}\right)=\nabla \eta^{\perp}=\left(-\partial \eta / \partial x_{2}, \partial \eta / \partial x_{1}\right) .
$$

At this level, the divergence-free condition on $\varphi$ is automatically satisfied and the steady equations take the form

$$
\int \nabla \nabla^{\perp} \eta: v \otimes v d x=-\int\left(\nabla^{\perp} \eta, f\right) d x
$$

The objective is to verify (3.6) for all $C^{2}$ functions $\eta$.

By appealing to the Fourier resolution

$$
\eta=\int e^{i(x, \xi)} \hat{\eta}(\xi) d \xi
$$

and a simple density argument, it is sufficient to prove (3.6) for all plane waves, i.e. functions of the form

$$
\eta=h\{(x, \xi)\}
$$

where $h$ depends on one variable. The strategy is to fix the normal $\xi$ and prove (3.6) for all $C^{2}$ functions $h$. 
In general orthogonal coordinates, the left side of (3.6) takes the form

$$
\iint \frac{\partial^{2} \eta}{\partial x_{1} \partial x_{2}}\left(v_{2}^{2}-v_{1}^{2}\right)+\left(\frac{\partial^{2} \eta}{\partial x_{2}^{2}}-\frac{\partial^{2} \eta}{\partial x_{1}^{2}}\right) v_{1} v_{2} d x_{1} d x_{2}
$$

We may choose coordinates so that $\xi=(1,0)$ in which case equation $(3.6)$ acting on plane waves of the form (3.7) reduces to

$$
\iint h^{\prime \prime}\left(x_{1}\right) v_{1} v_{2} d x_{1} d x_{2}=\iint h^{\prime}\left(x_{1}\right) f_{2} d x_{1} d x_{2} \text {. }
$$

In order to pass to the limit it suffices to construct a truncated test function which vanishes on the set where convergence is weak and then remove the truncation.

Fix $r>0$. Notice that $v_{\varepsilon}$ converges strongly to $v$ except on a set whose projection $I$ onto the $x_{1}$-axis has small Lebesgue measure: $I$ is a union of intervals $I_{j}$ with small total length:

$$
\sum\left|I_{j}\right| \leq \delta
$$

Here the set $I$ is the orthogonal projection of $F_{r}^{c}$ onto the $x_{1}$-axis.

Let $\tau$ denote the characteristic function of $I^{c}$ and consider special test functions of the form

$$
h=\int^{x} \int^{z} \tau(s) k^{\prime \prime}(s) d s d z
$$

where $k$ is an arbitrary $C^{2}$ function with compact support. Notice that the second derivative of $h$ vanishes on the set where convergence may be weak but not strong:

$$
h^{\prime \prime}=\tau k^{\prime \prime}
$$

for almost all points. Here $h$ is a $C^{1}$ function whose second derivative in the sense of distributions is represented by an $L^{\infty}$ function, namely $\tau k^{\prime \prime}$. Test functions with this degree of regularity are admissible in the weak formulation of the equations since the vector fields $v$ under consideration lie in $L^{2}:$ the associated quadratic terms such as $v_{1} v_{2}$ lie in $L^{1}$ and allow any $L^{\infty}$ multiplier. A simple approximation argument using the Lebesgue dominated convergence theorem confirms this fact.

Taking $h$ in this special truncated class yields the equation

$$
\iint \tau\left(x_{1}\right) k^{\prime \prime}\left(x_{1}\right) v_{1}^{\varepsilon} v_{2}^{\varepsilon} d x_{1} d x_{2}=\iint h^{\prime}\left(x_{1}\right) f_{2}^{\varepsilon} d x_{1} d x_{2} .
$$

Since $v_{\varepsilon}$ converges strongly to $v$ on the complement of the set where $\tau$ vanishes and since $f_{\varepsilon}$ converges weakly to $f$ it follows that

$$
\iint \tau k^{\prime \prime} v_{1} v_{2} d x_{1} d x_{2}=\int h^{\prime} f_{2} d x_{1} d x_{2} \text {. }
$$

The last step is to fix the function $k$ and take a sequence of functions $\tau_{j}$ which tend pointwise almost everywhere to the identity. In this case the right 
side of (3.10) converges to the integral of $k^{\prime} f_{2}$ since $h_{j}^{\prime}$ converges uniformly to $k^{\prime}$ :

$$
\lim h_{j}^{\prime}=\lim \int^{x} \tau_{j} k^{\prime \prime} d s=k^{\prime}(s),
$$

by Lebesgue's theorem. The left side of (3.10) converges to the integral of $k^{\prime \prime} u_{1} u_{2}$ and we conclude that

$$
\iint k^{\prime \prime} v_{1} v_{2} d x=\int k^{\prime} f_{2} d x
$$

for all $C^{2}$ functions $k$ with compact support. Thus (3.6) holds for all $C^{2}$ functions of the form $\eta=k\{(x, \xi)\}$.

The existence of the characteristic functions $\tau_{j}$ approaching the identity pointwise a.e. follows from the structure of the projection $I(r)$ of the exceptional set $F_{r}^{c}$ onto the $x_{1}$-axis. We simply take a sequence of values $r_{j}$ tending to zero: the one-dimensional Lebesgue measure of $I\left(r_{j}\right)$ tends to zero since it is bounded by $c r_{j}^{1-\gamma}$; the characteristic function of the complement tends to zero pointwise a.e.

A similar argument using plane waves in $R^{2} \times R$ leads to a proof of Theorem 2 stated in the Introduction dealing time-dependent concentration-cancellation. Proof of Theorem 2. We consider the distributional form of the equations, namely

$$
\iint\left(\varphi_{t}, v\right)+\nabla \varphi: v \otimes v d x d t=0
$$

where $\operatorname{div} \varphi=0$ and express the test field $\varphi$ in terms of a scalar potential $\eta$

$$
\varphi=\nabla^{\perp} \eta=\left(-\partial \eta / \partial x_{2}, \partial \eta / \partial x_{1}\right)
$$

Here $\eta=\eta(y)$ and $y=(x, t)$. In terms of $\eta$ the equation becomes

$$
\iint\left(\nabla^{\perp} \eta_{t}, v\right)+\nabla \nabla^{\perp} \eta: v \otimes v d x d t=0
$$

Since $v^{\varepsilon}$ converges weakly to $v$ in $L^{2}$, we have

$$
\lim _{\varepsilon \rightarrow 0} \iint\left(\nabla^{\perp} \eta_{t}, v^{\varepsilon}\right) d x d t=\iint\left(\nabla^{\perp} \eta_{t}, v\right) d x d t
$$

if $\nabla^{\perp} \eta_{t}$ lies in $L^{2}$.

As in the proof of Theorem 3.1 we shall appeal to the Fourier transform to reduce the problem to verifying (3.12) for plane wave test functions, i.e. functions of the form

$$
\eta=h(y \cdot \xi)=h\left(t \xi_{0}+x_{1} \xi_{1}+x_{2} \xi_{2}\right) .
$$

We remark that if both $\xi_{1}$ and $\xi_{2}$ vanish, then $\eta$ depends only on $t$ and the equation (3.12) holds as a consequence of $L^{2}$ weak convergence (3.13). Thus, 
selecting appropriate coordinates we may assume without loss of generality that $\xi_{1}=1$ and $\xi_{2}=0$.

As in the proof of Theorem 3.1 we shall verify (3.12) for potentials $\eta$ of the form (3.14) where

$$
h=\int^{x} \int^{z} \tau(s) k^{\prime \prime}(s) d s d z
$$

$k$ is an arbitrary $C^{2}$ function and $\tau$ is a bounded approximation of the identity. Fixing $k$ and letting $\tau$ approach the identity yields (3.12) for all $\eta$ of the form (3.14) with $h$ replaced by $k$. This is the desired result. The verification of this outline proceeds as follows.

In the aforementioned coordinates we have

$$
\begin{aligned}
\iint \nabla \nabla^{\perp} \eta: v^{\varepsilon} \otimes v^{\varepsilon} d x d t & =\iiint \frac{\partial^{2} \eta}{\partial x_{1}^{2}} v_{1}^{\varepsilon} v_{2}^{\varepsilon} d x_{1} d x_{2} d t \\
& =\iiint \tau\left(t \xi_{0}+x_{1}\right) k^{\prime \prime}\left(t \xi_{0}+x_{1}\right) v_{1}^{\varepsilon} v_{2}^{\varepsilon} d x_{1} d x_{2} d t
\end{aligned}
$$

By hypothesis the Hausdorff dimension of the space-time exceptional set is less than one. Thus $v^{\varepsilon}$ converges to $v$ in $L^{2}$ on the complement of a set whose space-time (orthogonal) projection $I$ onto the line through $\left(\varepsilon_{0}, 1,0\right)$ has arbitrarily small Lebesgue measure. Choosing $\tau$ to be the characteristic function of $I^{c}$ as in the proof of Theorem 3.1 shows that

$$
\lim \iint \nabla \nabla^{\perp} \eta: v^{\varepsilon} \otimes v^{\varepsilon} d x d t=\iint \nabla \nabla^{\perp} \eta: v \otimes v d x d t
$$

for functions $\eta$ of the form (3.14) and (3.15). Combining this statement with (3.13) yields the basic relation (3.12) for all such $\eta$. Since $\tau$ may be taken arbitrarily close to the identity we deduce that (3.12) holds for all $\eta$ of the form $\eta=k(y \cdot \xi)$ where $k$ is an arbitrary $C^{2}$ function.

Remark 1. The analysis of the steady problem above does not imply that the form

$$
S\left(\varphi, u_{\varepsilon}\right)=\int \nabla \varphi: v_{\varepsilon} \otimes v_{\varepsilon} d x
$$

is continuous in the weak topology of $L^{2}$ if $\varphi$ is smooth and divergence-free. If the test function is smooth and fixed the limiting process may produce a value which differs from $S\left(\varphi, v_{\varepsilon}\right)$. However, we observe in this connection that if the weak-star defect measure is concentrated inside a set with Hausdorff dimension less than one, then $S$ as well as its time-dependent counterpart $T$ are continuous in the weak $L^{2}$ topology:

$$
\lim S\left(\varphi, v_{\varepsilon}\right)=S(\varphi, v)
$$

if $\mu$ is concentrated inside a set $E$ such that $H^{\gamma}(E)<\infty$ for some $\gamma<1$. The proof follows the lines of Theorem 3.1. 
Remark 2. In general the statement that the weak-star defect measure is concentrated on a small set is stronger than the corresponding statement for the reduced defect measure. Nevertheless $u$ satisfies the equation although $u$ is not accessible through the sequence $v_{\varepsilon}$ from the viewpoint of $S$ and $T$ and the classical weak topology. A progressive renormalization of the topology can suppress weak-star defects: a slight variation on the arguments above shows that there exists a sequence of smooth fields $\varphi_{\varepsilon}$ tending to $\varphi$ such that

$$
\lim S\left(\varphi_{\varepsilon}, v_{\varepsilon}\right)=S(\varphi, v)
$$

although it may happen that

$$
\lim S\left(\varphi, v_{\varepsilon}\right) \neq S(\varphi, v)
$$

in general. A similar statement holds for $T$.

\section{UNIFORM DISTRIBUTION FUNCTIONS}

Our goal here is to prove the uniformization theorem described earlier in the Introduction. We begin our discussion with several preliminary remarks and then we state this theorem. The proof of this theorem is completed in this section except for results which are a corollary of the Chebyshev inequality, described in the next section.

Let $\omega$ denote a nonnegative Borel measure on $R^{n}$ with compact support. In this section we are concerned with the behavior of the radial distribution function of $\omega$ :

$$
\omega(x, s)=\omega\{B(s, x)\}
$$

where $B$ denotes the open ball of radius $s$ centered at $x$.

We first remark that $\omega$ decays at most points of the space $R^{n}$. Fix positive numbers $\delta$ and $r$ and consider the closed set $E$ on which $\omega$ decays algebraically of order $\delta$ :

$$
E=\left\{x: \omega(s, x) \leq s^{\delta} \text { if } 0 \leq s \leq r\right\} .
$$

The exceptional set $E^{c}$ on which decay is lost has finite $\delta$-order Hausdorff premeasure:

$$
H_{r}^{\delta}\left(E^{c}\right) \leq \text { const. } T M \omega
$$

(see Lemma 4.1 below). Thus, the weight which $\omega$ assigns to a ball of radius $s$ decays locally like $s$ except possibly for a set with "dimension" equal to $\delta$. The size of the exceptional set is bounded in terms of the total mass of $\omega$ and a constant that depends only on $\delta$. The proof relies on a standard covering lemma.

A general problem deals with the existence of uniform exceptional sets. Given a family of Borel measures with uniformly bounded total mass, does there exist a sequence all of whose members exhibit prescribed decay on the complement of a small set? We shall answer this question in the affirmative through a study of the associated Riesz potentials. Without loss of generality we shall assume that all distributions under discussion are supported inside the unit ball. 
The existence of uniform exceptional sets for uniformly bounded measures is established in the following theorem.

Theorem 4.1. Consider a family of nonnegative measures $\omega$ on $R^{n}$ satisfying $T M \omega \leq 1$. Fix parameters $\delta$ and $\gamma$ such that $\delta>0$ and $\gamma>n \delta$. There exists a sequence $\omega_{k}$ with the following property. For every positive $r$ there exists a closed set $F_{r}$ such that

$$
F_{r} \subset\left\{x: \omega_{k}(s, x) \leq K s^{\delta}, 0 \leq s \leq 1, k \geq 1 / r\right\}
$$

and the $\gamma$-order Hausdorff premeasure of level $r$ satisfies

$$
H_{r}^{\gamma}\left(F_{r}^{c}\right) \leq c(\delta, \gamma)
$$

where

$$
K=c+c r^{-\delta}
$$

and $c$ is a universal constant.

Remark 1. The smaller the value of $r$ the farther along the sequence it is necessary to go in order to achieve uniformity in decay of the distribution function. The choice of $k \geq 1 / r$ is taken for convenience.

Remark 2. The premeasure of the uniform exceptional set $F_{r}^{c}$ is bounded by a constant which depends only on $\delta$ and $\gamma$. The restriction of $\gamma$ to values greater than $n \delta$ is adopted for simplicity so that the growth of the coefficient $K$ in (4.2) is described by the same parameter as the decay of the distribution function, namely $\delta$. Similar results hold for arbitrary $\gamma$.

Several preliminary remarks are in order. We recall that the weight which $\gamma$-order Hausdorff measure assigns to a set is expressed through a limit of premeasures:

$$
H^{\gamma}(E)=\lim _{r \rightarrow 0} H_{r}^{\gamma}(E)=\sup _{r>0} H_{r}^{\gamma}(E) .
$$

The premeasure $H_{r}^{\gamma}(E)$ is obtained by considering the most efficient countable cover of $E$ by open balls $B_{j}$ and adding their radii $r_{j}$ to the specified power $\gamma$ :

$$
H_{r}^{\gamma}(E)=\inf \left\{\sum r_{j}^{\gamma}: E \subset \cup B_{j}, r_{j} \leq r\right\} .
$$

In general $H_{r}^{\gamma}$ is subadditive. In the limit as the level of observation $r$ approaches zero, only the fine covers enter into the countably additive measure $H^{\gamma}$.

More generally, a Hausdorff measure $H^{\beta}$ may be defined through limits of premeasure $H_{r}^{\beta}$ based on an arbitrary continuous increasing function $\beta$ vanishing at the origin:

$$
H_{r}^{\beta}(E)=\inf \left\{\sum \beta\left(r_{j}\right)\right\} .
$$

For technical reasons associated with covering arguments it is convenient to assume $\beta(5 s) \leq K \beta(s)$ for some constant $K$. 
Lemma 4.1. Suppose $\omega$ is a nonnegative measure with finite total mass. The closed set

$$
E^{c}=\{x: \omega(s, x) \leq \beta(s) \text { if } 0 \leq s \leq r / 5\}
$$

satisfies

$$
H_{r}^{\beta}(E) \leq K T M \omega
$$

Proof. Standard continuity properties of measures imply that $E^{c}$ is closed. In order to verify (5.2), we observe that

$$
E=\{x: \omega(s, x)>\beta(s) \text { for some } s \text { with } 0 \leq s \leq r / 5\}
$$

can be covered by a union of balls centered at points in $E$ :

$$
E \subset \bigcup\{B(s(x), x): x \in E, 0 \leq s(x) \leq r / 5\} .
$$

A standard covering lemma stated below implies there exists a countable number of points $x_{j}$ in $E$ such that the sequence

$$
B_{j}=B\left(s\left(x_{j}\right), x_{j}\right)
$$

is disjoint and covers $E$ after a five-fold expansion:

$$
E \subset \bigcup B_{j}^{*}, \quad B_{j}^{*}=B\left(5 s\left(x_{j}\right), x_{j}\right) .
$$

Summing the inequalities

$$
\beta\left(5 s_{j}\right) \leq K \beta\left(s_{j}\right) \leq K \omega\left(B_{j}\right)
$$

yields

$$
\sum \beta\left(5 s_{j}\right) \leq K \sum \omega\left(B_{j}\right)=K \omega\left\{\cup B_{j}\right\} \leq K T V \omega
$$

since $B_{j}$ are disjoint. Thus $E$ can be covered by a countable union of balls, namely $B_{j}^{*}$, whose radii $5 s_{j}$ are summable with respect to $\beta$ and do not exceed the prescribed level $r$. Inequality (4.4) follows from definition 4.3.

Covering Lemma. If $J$ is an arbitrary family of balls $B_{\alpha}$ contained in a bounded set of $R^{n}$ then there exists a countable disjoint collection $B_{r_{j}}$ of $J$ whose five-fold expansion covers $J$ :

$$
\bigcup_{J} B_{\alpha} \subset \bigcup B_{5 r_{j}}
$$

We refer the reader to [8] for a proof.

With this preliminary information, we next describe the proof of the uniformization theorem.

Proof. The theorem follows as a corollary of a similar statement that establishes a uniform pointwise bound on the associated Riesz potentials,

$$
\varphi(x)=\int_{R^{n}} \frac{1}{|x-y|^{\delta}} d \omega(y) .
$$


We shall show that there exist large closed sets $F_{r}$ such that

$$
\varphi_{k}(x) \leq K(r) \text { for } k \geq 1 / r
$$

if $x \in F_{r}$. It follows that

$$
s^{-\delta} \omega_{k}(s, x) \leq \int_{B(s, x)} \frac{1}{|x-y|^{\delta}} d \omega_{k} \leq \varphi_{k}(x) \leq K .
$$

More refined results can be obtained using more general kernels.

The strategy is to show that the associated Riesz potentials $\varphi$ lie in a compact subset of $W^{1, p}$ if $p<n /(\delta+1)$. We establish this fact in the remainder of this section. To complete the proof of the theorem we use the following lemma, which is a corollary of the Chebyschev inequality established in the next section. The proof of this lemma is postponed until that section.

Lemma 4.2. Assume that $f_{k} \rightarrow f$ in $W^{1, p}\left(R^{n}\right)$ with $1<p<n$. There exists a subsequence so that for every positive $\varepsilon$ there exists a Borel set $P$ such that $f_{k}$ converges pointwise to $f$ on $P$ and $H^{\gamma}\left(P^{c}\right)=0, \gamma=n-p+\varepsilon$. Furthermore, $P$ is an increasing limit of sets $P_{j}$ on which $f_{k}$ converges uniformly to $f$. The $\gamma$-order premeasure of $P_{j}$ tends to zero. More precisely,

$$
\begin{aligned}
& P=\bigcup P_{j}, \quad P_{j+1} \supset P_{j}, \\
& H_{r_{j}}^{\gamma}\left(P_{j}^{c}\right) \leq a_{j}
\end{aligned}
$$

where $a_{j}, r_{j}$ tend to zero.

Using the compactness of the Riesz potentials $\varphi_{k}$ to be established below, we complete the proof. We apply Lemma 4.2 to $\varphi_{k}$. Thus, there exists a subsequence $\varphi_{k}$ and a function $\varphi$ in $W^{1, p}$ so that $\varphi_{k}$ converges to $\varphi$ on the complement of a set with bounded $\gamma$-order premeasure. Since the limit potential $\varphi$ belongs to $W^{1, p}\left(R^{n}\right), \varphi$ has a pointwise bound on the complement of a set with bounded $\gamma$-order premeasure (see the main Chebyschev inequality in the next section and [5]). Combining the two exceptional sets provides a uniform pointwise bound (4.5) on a sequence of potentials $\varphi_{k}$ where $F_{r}$ satisfies (4.1).

The compactness of the potentials $\varphi$ follows from the hypothesized uniform bound on the total mass of the measures $\omega$.

If $D^{s}$ denotes the operator defined through the Fourier transform by

$$
\hat{D}^{s} \varphi=|\xi|^{s} \hat{\varphi}
$$

then

provided that $\delta+s<n$.

$$
D^{s} \varphi=\int \frac{1}{|x-y|^{\delta+s}} d \omega(y)
$$

Indeed, on the Fourier side we have

$$
\hat{D}_{s} \varphi=|\xi|^{s} \hat{\varphi}=c|\xi|^{s}|\xi|^{-n+\delta} \hat{\omega}(\xi)=c|\xi|^{-n+(\delta+s)} \hat{\omega}(\xi),
$$


since

$$
|\hat{y}|^{-\theta}=c|\xi|^{\theta-n} \quad \text { if } 0<\theta<n .
$$

Since all of the measures $\omega$ are supported inside a fixed compact set, namely the unit ball, Jensen's inequality implies

$$
\left|D^{s} \varphi(x)\right|^{p} \leq c \int \frac{1}{|x-y|^{(\delta+s) p}} d \omega(y) .
$$

Integration with respect to $x$ yields a uniform bound of the form

$$
\int_{R^{n}}\left|D^{s} \varphi\right|^{p} d x \leq c \iint \frac{1}{|x-y|^{(\delta+s) p}} d x d \omega \leq c
$$

if $(\delta+s) p<n$.

As discussed in the Appendix above (A-12), for the purpose of obtaining the uniform estimates on the potentials in (4.5) when $\omega$ has bounded support, we can use the Bessel potentials defined in (A-12) (see Stein [8]) in an equivalent fashion as we used the Riesz potentials above. The identical argument given above applies and we obtain a uniform bound on the Bessel potentials $\varphi$ of order $\delta$ in $L^{t, p}$ for any $t$ with $(\delta+t) p<n$. Here $L^{t, p}$ is the space of tempered distributions $u$ so that $\left(1+|\xi|^{2}\right)^{t / 2} u(\hat{\xi})$ has an inverse Fourier transform in $L^{p}\left(R^{n}\right)$.

The injection, $L^{t, p} \rightarrow W^{t-\varepsilon, p}$, is continuous for any $\varepsilon>0$ and the injection $W^{t, p} \rightarrow W^{s, p}$ is compact for $t>s[1,8]$. Thus, if we consider uniformly bounded measures supported inside a fixed ball, the estimate above proves that the associated Riesz potentials restricted to any bounded subset of $R^{n}$ lie in a compact subset of $W^{s, p}$ provided that $(\delta+s) p<n$. Since for $n=2, s=1$ satisfies these restrictions, the proof is complete.

\section{Chebyshev inequalities}

Our objective here is to establish the result on uniform convergence of functions $W^{1, p}\left(R^{n}\right), p<n$, which we stated in Lemma 4.2. Also, in order to complete the proof of the uniformization theorem of the last section, we need to prove that an arbitrary function $f$ in $W^{1, p}\left(R^{n}\right)$ is uniformly bounded except for a set with small $\gamma$-order Hausdorff premeasure with $\gamma=n-p+\varepsilon$. Both of these results are corollaries of a refinement of the classical Chebyshev inequality which we develop here. This refinement is similar to the Chebyshev inequality from [5] except here we replace $p$-capacity by an appropriate Hausdorff premeasure. We also present a logarithmic refinement.

The classical Chebyshev inequality states that the Lebesgue distribution function $\sigma$ of an element $f$ of $L^{p}\left(R^{n}\right)$ is dominated by an algebraic function of order $p$ :

$$
\sigma(\lambda)=H^{n}\{x:|f| \geq \lambda\} \leq \lambda^{-p} \int|f|^{p} d x
$$

For functions with compact support, the essential behavior is associated with large values of $\lambda$. 
If $f$ lies in $W^{1, p}$ then $n$-dimensional Lebesgue measure can be replaced in (5.1) by a finer set function, namely $\gamma$-order Hausdorff premeasure where $\gamma=n-p+\varepsilon$ and $\varepsilon$ is arbitrarily small.

Theorem 5.1. If $\varepsilon>0$ and $p<n$ then there exists a constant $c=c(n, p, \varepsilon)$ such that

$$
H_{r(\theta)}^{\gamma}\{x:|f| \geq \lambda\} \leq c \theta^{p}
$$

for all $f$ in $W^{1, p}\left(R^{n}\right)$ where

$$
\begin{aligned}
& \theta=\alpha / \lambda, \quad \alpha=\left\{\int_{R^{n}}|\nabla f|^{p} d x\right\}^{1 / p}, \\
& r(\theta)=c \theta^{q / n}, \quad q=n p / n-p .
\end{aligned}
$$

Remark 1. The detailed structure of the level function $r(\theta)$ is not crucial for the applications to the Euler equations. We shall make use only of the fact that $r$ tends to zero for fixed $\lambda$ as $\alpha$ tends to zero and for fixed $\alpha$ as $\lambda$ tends to infinity. This is due essentially to the fact that premeasure depends monotonically on the level:

$$
H_{r}^{\gamma}(E) \leq H_{s}^{\gamma}(E) \text { if } r>s .
$$

Remark 2. In treating discontinuous functions $f$ it is convenient to replace $f$ by the corresponding Lebesgue function $L f$ : if $x$ is a Lebesgue point of $f$ then $L f(x)$ is the associated Lebesgue value, otherwise $L f$ is zero. We shall adopt this convention throughout the paper.

Remark 3. If $f$ lies in $W^{1, p}\left(R^{n}\right), p<n$, then the complement of the Lebesgue set has vanishing $H^{\gamma}$ measure, $\gamma=n-p+\varepsilon$ for all positive $\varepsilon$ [5].

Proof. We claim that the interval set

$$
A=\{x:|f| \geq \lambda\}
$$

is contained in a set of the form

$$
D=\left\{x: r^{-\gamma} \int_{B(r, x)}|\nabla f|^{p} d y \geq M \text { for some } r \text { with } 0<r \leq r_{0}\right\}
$$

if the parameters $r_{0}$ and $M$ are chosen appropriately. In short, $A$ is contained in a set where the gradient fails to exhibit uniform local decay of a specific order: the normalized local maximal function of the gradient remains above $M$ for some ball of radius $r \leq r_{0}$. The choice of $r_{0}$ and $M$ are given below.

A standard argument using the covering lemma of the previous section shows that

$$
H_{5 r_{0}}^{\gamma}(D) \leq \text { const. } M^{-1} \int|\nabla f|^{P} d y
$$

with a universal constant. Since $A$ is contained in $D$ a similar inequality holds for $A$ and yields the desired result. The proof of the inclusion is based upon the standard fact that the pointwise values of a function are dominated by the 
normalized maximal function of the derivative. At a Lebesgue point $f$ may be expressed as a telescoping sum of dyadic local averages:

$$
f(x)=f_{0}(x)+\sum_{j \geq 1} f_{j+1}(x)-f_{j}(x)
$$

where

$$
f_{j}=f_{B_{j}} f(y) d y
$$

and $B_{j}=B_{j}\left(r_{j}, x\right), r_{j}=2^{-j} r_{0}$. The slash denotes integration with respect to normalized Lebesgue measure

$$
f_{E} f d y=\frac{1}{m(E)} \int_{E} f d y
$$

An application of the Poincaré inequality reveals the dependence on the local behavior of the derivative:

$$
|f| \leq\left|f_{0}\right|+2^{n} \sum r_{j}\left\{f_{B_{j}}|\nabla f|^{p} d y\right\}^{1 / p},
$$

since the difference of the two consecutive averages is bounded by the average oscillation,

$$
\left|f_{j+1}-f_{j}\right| \leq 2^{n} f_{B_{j}}\left|f-f_{j}\right| d y .
$$

Suppose that $A$ is not contained in $D$ and choose a Lebesgue point $z$ in $A$ such that

$$
f_{B(r, z)}|\nabla f|^{p} d y \leq M r^{-p+\varepsilon} \text { if } 0<r \leq r_{0} .
$$

It follows that

$$
\lambda \leq\left|f_{0}(z)\right|+2^{n} M^{1 / p} \sum r_{j}^{\varepsilon / p}
$$

since $z$ lies in $A$ and $r_{j} \leq r_{0}$. The strategy is to select $M$ and $r_{0}$ so that (5.3) is violated. For this purpose the first term on the right of (5.2) can be estimated using the Sobolev inequality:

$$
\begin{aligned}
\left|f_{0}(z)\right| & =\left|f_{B_{0}} f d y\right| \leq\left\{f_{B_{0}}|f|^{q} d y\right\}^{1 / q} \\
& \leq r_{0}^{-n / q}\left\{\int_{R^{n}}|f|^{q} d y\right\}^{1 / q} \leq c_{1} r_{0}^{-n / q} \alpha,
\end{aligned}
$$

where $c_{1}$ is a universal constant and $B_{0}=B\left(r_{0}, z\right)$. Letting

$$
c_{2}=2^{n} \sum_{j} r_{j}^{\varepsilon / p}
$$

yields the inequality

$$
\lambda \leq c_{1} r_{0}^{-n / q} \alpha+c_{2} M^{1 / p}
$$


as a consequence of (5.3). We conclude that $A$ is contained in $D$ if $r_{0}$ and $M$ are chosen so that each term of the right side of (5.4) equals $\lambda / 3$ :

$$
r_{0}=\left(3 c_{1} \theta\right)^{q / n}, \quad M=\left(3 c_{2}\right)^{-p} \lambda^{p} .
$$

The value of $c$ in the conclusion of the theorem is easily derived from $c_{1}$ and $c_{2}$.

One of the consequences of the generalized Chebyshev inequality is the following refinement of Egoroff's theorem, which we used in Lemma 4.2.

Theorem 5.2. Suppose $f_{i}$ converges to $f$ in $W^{1, p}\left(R^{n}\right), p<n$. Then there exists a subsequence $f_{k}$ with the following properties. For every positive $\varepsilon$ there exists a Borel set $P$ such that $f_{k}$ converges pointwise to $f$ on $P$ and $H^{\gamma}\left(P^{c}\right)=$ $0, \gamma=n-p+\varepsilon$. Furthermore, $P$ is an increasing limit of sets $P_{j}$ on which $f_{k}$ converges uniformly to $f$. The $\gamma$-order premeasure of $P_{j}$ tends to zero. More precisely,

$$
P=\bigcup P_{j}, \quad P_{j+1} \supset P_{j}, \quad H_{r_{j}}^{\gamma}\left(P_{j}\right) \leq a_{j}
$$

where $a_{j}$ and $r_{j}$ are certain specified sequences.

Remark 1. As mentioned above, pointwise values refer to the associated Lebesgue functions. The associated Lebesgue functions converge uniformly on the complement of a small set.

Remark 2. The rate at which $a_{j}$ and $r_{j}$ in (5.5) tend to zero depends on the manner of indexing. We shall take $a_{j}=1 / j$ for convenience.

Proof. Consider the deviation set $A_{s, k}$ at level $s$ and tolerance $1 / k$ :

$$
A_{s, k}=\left\{x:\left|f_{s}(x)-f(x)\right| \leq 1 / k\right\} \text {. }
$$

A uniform deviation set $D_{m, k}$ for indices above $m$ is defined by intersection:

$$
D_{m, k}=\bigcap_{s \geq m} A_{s, k}
$$

A typical uniform convergence set takes the form

$$
U=\bigcap_{k \geq k_{0}} D_{m(k), k}
$$

for some integer-valued function $m(k)$. The definitions imply that $f_{k}$ converges uniformly to $f$ on $U$. The problem is to estimate the complement:

$$
U^{c}=\bigcup_{k \geq k_{0}} F_{k}, \quad F_{k}=\bigcup_{s \geq m(k)} A_{s, k}^{c} .
$$

Taking

$$
\alpha_{m}^{p}=\int\left|\nabla f_{m}-\nabla f\right|^{p} d y
$$


and $\lambda=1 / k$ in the generalized Chebyshev inequality yields the following bound on a typical term in (5.5):

$$
H_{r_{s}}^{\gamma}\left(A_{s, k}^{c}\right) \leq c k^{p} \alpha_{s}^{p}
$$

where $r_{s}=r\left(k \alpha_{s}\right)$. By passing to a subsequence we may assume that the sequence $\alpha_{m}$ is nonincreasing. Consequently,

$$
H_{r_{m}}^{\gamma}\left(A_{s, k}^{c}\right) \leq H_{r_{s}}^{\gamma}\left(A_{s, k}^{c}\right) \leq c k^{p} \alpha_{s}^{p}
$$

if $s \geq m$ by virtue of the monotonicity properties of premeasure. Notice that $r_{m} \geq r_{s}$.

Subadditivity yields an upper bound on the union of complements,

$$
H_{r_{m}}^{\gamma}\left(\bigcup_{s \geq m} A_{s, k}^{c}\right) \leq c k^{p} \sum_{s \geq m} \alpha_{s}^{p} .
$$

By passing to a subsequence we may assume that

$$
\sum \alpha_{s}^{p}<\infty
$$

and then choose a function $m(k)$ so that the right side of (5.7) is summable, e.g.,

Therefore

$$
k^{p} \sum_{s \geq m(k)} \alpha^{p} \leq 1 / k^{2} .
$$

$$
H_{r_{k}}^{\gamma}\left(F_{k}\right) \leq c / k^{2}
$$

where $r_{k}=r\left(k \alpha_{m(k)}\right)$. By passing to a subsequence we may assume that $r_{k}$ is nonincreasing and hence that

$$
H_{r_{j}}^{\gamma}\left(F_{k}\right) \leq H_{r_{k}}^{\gamma}\left(F_{k}\right) \leq c / k^{2}
$$

Fixing $j$ and summing on $k$ yields the desired result

by defining

$$
H_{r_{j}}^{\gamma}\left(\bigcup_{k \geq j} F_{k}\right) \leq c \sum_{k \geq j} 1 / k^{2} \leq c / j
$$

$$
P_{j}^{c}=\bigcup_{k \geq j} F_{k} .
$$

Remark 1. The proof provides a criterion to select a subsequence with the desired uniform convergence properties, namely the summability condition (5.8) together with associated monotonicity conditions. The existence of a selection principle is useful in the analysis of defects in the time dependent Euler equations.

In order to obtain the logarithmic refinement on the size of the exceptional set it is necessary to extend the Chebyshev inequality to more general convex functions as follows.

Let $g$ be an increasing convex function on $[0, \infty)$ vanishing at the origin and $m$ a positive integer. 
Theorem 5.3. Suppose $h$ is a positive decreasing continuous function on $(0, \infty)$ such that

$$
\int_{1}^{\infty} h\left(e^{-s}\right) d s \leq 1
$$

If $\gamma=s^{n} g\left\{s^{-m} h(s)\right\}$ then Hausdorff premeasure based on the function $\gamma$ satisfies the inequality

$$
H_{r}^{\gamma}\{x:|f| \geq \lambda\} \leq c \int g\left\{\left|D^{m} f\right| / \lambda\right\} d y
$$

for all smooth compactly supported functions $f$ on $R^{n}$. The level $r$ is defined by

$$
r^{n}=c \lambda^{-1} \int|f| d y
$$

and $c$ is a universal constant.

Remark 2. The right side of (5.9) may be replaced by an arbitrary positive constant. The change influences only the choice of $c$.

Remark 3. The inequality (5.10) extends by closure to elements of the associated Orlicz-Sobolev space.

Remark 4. The rate at which the level $r$ approaches zero as $\lambda$ tends to infinity may be improved. The refinement is not required for the present applications to the Euler equations.

Proof. Take $\lambda=1$ and consider $A=\{x:|f| \geq 1\}$. We claim that

$$
A \subset D=\left\{x: \int_{B(s, x)} g\left(\left|D^{m} f\right|\right) d y \leq c \gamma(s) \text { for some } 0<s \leq n\right\} .
$$

The dyadic representation (5.2) yields

$$
1 \leq|f(x)| \leq\left|f_{0}(x)\right|+2^{n} \sum r_{j}^{m} f_{B_{j}}\left|D^{m} f\right| d y
$$

if $x$ lies in $A$. Jensen's inequality implies that

$$
1 \leq\left|f_{0}\right|+2^{n} \sum r_{j}^{m} \sigma_{j}
$$

where

$$
\sigma_{j}=g^{-1}\left\{f_{B_{j}} g\left(\left|D^{m} f\right|\right) d y\right\} .
$$

Suppose $x$ does not lie in $D$ and choose the parameters so that each term on the right of $(5.11)$ does not exceed $1 / 3$. The first term is easily handled:

$$
\left|f_{0}\right| \leq f_{B(r, x)}|f| d y \leq c r^{-n} \int|f| d y .
$$

The second term admits an upper bound of the form

$$
2^{n} \sum h\left(r_{j}\right)=2^{n} \sum h\left(2^{-j} r\right)
$$


since $x$ is in $D^{c}$ and $\sigma_{j} \leq r_{j}^{-m} h\left(r_{j}\right)$. Thus it suffices to consider decreasing functions $h$ such that

$$
\int_{1}^{\infty} h\left(2^{-s}\right) d s \leq c
$$

where $c$ is an appropriate constant. The dyadic sequence $r_{j}=r 2^{-j}$ may be replaced by any exponential sequence and the constant in (5.12) may be normalized to unity.

Remark 5. Theorem 5.3 is the key ingredient in the proof of the logarithmic refinement on the size of exceptional sets for Poisson sequences mentioned in the Introduction.

\section{6. $L^{p}$ VELOCITY ESTIMATES}

In the last two sections, we developed a uniformization theorem which yields a uniform decay rate for the radial distribution functions of vorticity, $\omega(s, x)=$ $\omega\{B(s, x)\}$, on a closed set with complement having small Hausdorff premeasure. Here we shall prove that if the radial distribution function $\omega$ decays with order $\delta$ on a set $F$, then the velocity field $v=\nabla^{\perp} \psi$ admits a uniform $L^{p}$ bound on the set $F$ for some $p$ with $p>2$. This fact combined with the discussion presented in the Introduction and $\S 4$ yields Theorem 3 on Hausdorff dimension of the reduced defect for elliptic sequences stated in the Introduction.

Without loss of generality, we assume that the stream function $\psi$ satisfying $\Delta \psi=\omega$ has support in the unit ball (see the Appendix). We will prove that if the radial distribution function, $\omega(s, x)=\omega\{B(s, x)\}$, of the measure $\omega$ decays on a set $F$ with order $\delta$, then the velocity field $v=\nabla^{\perp} \psi$ lies in the Marcinkiewicz space $M^{p}$ where $p=2+\delta /(1-\delta)$, i.e.

$$
\sigma_{F}(\lambda) \equiv H^{2}\{x \in F:|v|>\lambda\} \leq c \lambda^{-p} .
$$

As usual, $v$ denotes the orthogonal complement of the gradient of $\psi$ :

$$
u=\nabla^{\perp} \psi=\int \frac{(x-y)^{\perp}}{|x-y|^{2}} d \omega(y) .
$$

Theorem 6.1. Suppose $\omega$ is a Borel measure on $R^{2}$ such that $T M \omega \leq 1$. If for all $x \in F$,

$$
\omega(s, x) \leq K s^{\delta} \quad \text { for } 0 \leq s \leq 1
$$

then

$$
\lambda \sigma_{F}(\lambda)^{1 / p} \leq c \delta^{-1}+c K
$$

where $c$ is a universal constant.

In other words, the $L^{\infty}$ norm of the normalized maximal function of the vorticity dominates the $M_{F}^{p}$ norm of the velocity:

$$
M_{F}^{p}(v) \leq c \delta^{-1}+c \sup _{x \in F} \Gamma_{\delta}(x, \omega)
$$


where

$$
\begin{gathered}
M_{F}^{p}(v)=\sup \lambda \sigma_{F}(\lambda)^{1 / p}, \\
\Gamma_{\delta}(x, \omega)=\sup _{s} s^{-\delta} \omega(s, x) .
\end{gathered}
$$

Remark 1. If $\omega$ is an arbitrary measure with finite total mass on $R^{2}$ then $v$ lies in $L^{p}$ for all $p<2$. An application of Jensen's inequality to (6.1) shows that

$$
|v|_{p} \leq c_{p} T M \omega
$$

The constant $c_{p}$ approaches infinity as $p$ approaches the value 2 .

Remark 2. $M_{F}^{p}$ is boundedly embedded in $L^{q}(F)$ if $p>q$ since

$$
\int|f|^{q} d x=-\int_{0}^{\infty} \lambda^{q} d \sigma=\int_{0}^{\infty} q \lambda^{q-1} \sigma(\lambda) d \lambda
$$

so the weak estimate yields $L^{q}$ bounds for $2<q<p$.

Proof. We shall derive pointwise bounds on $u$ over sets $A_{k}$ of the form

$$
A_{k}=\left\{x: \omega(s, x) \leq k s^{1+\varepsilon} \text { if } 0 \leq s \leq 1 / k\right\}
$$

and examine their growth with $k$ in comparison to the size of $A_{k}$. For this purpose we assume for simplicity that $\psi$ is smooth. The general case is easily treated by approximation.

For arbitrary $x$ we have

$$
|u(x)| \leq c \int|x-y|^{-1} d \omega=c \int_{0}^{1} s^{-1} d \omega(s, x) .
$$

If $x$ lies in $A_{k} \cap F$, an integration by parts is permissible in (6.3) to yield

$$
|u(x)| \leq c \omega(1, x)+c \int_{0}^{1} s^{-2} \omega(s, x) d x .
$$

Splitting the integral at $1 / k$ and using the global $\delta$-order decay (6.2) yields

$$
|u(x)| \leq c+c k \int_{0}^{1 / k} s^{-1+\varepsilon} d s+c \int_{1 / k}^{1} K s^{-2+\delta} d s .
$$

The optimal choice of $\varepsilon$, namely $\varepsilon=\delta$, produces the following pointwise bound on $A_{k}$ :

$$
|u(x)| \leq c+d k^{1-\delta}
$$

where $d=c \delta^{-1}+c K$.

If we choose $k$ so that

$$
2 c+d k^{1-\delta}=\lambda
$$

then $\{x \in F:|u|>\lambda\} \subset A_{k}^{c}$ and consequently

$$
\sigma_{F}(\lambda)=H^{2}\{x \in F:|u|>\lambda\} \leq H^{2}\left(A_{k}^{c}\right) .
$$


Thus the problem reduces to an estimate on the two-dimensional Lebesgue measure of $A_{k}^{c}$. From the definition it follows immediately that

$$
H_{5 / k}^{1+\delta}\left(A_{k}^{c}\right) \leq c / k
$$

using the covering lemma of $\S 5$. Thus $A_{k}^{c}$ can be covered by a countable union of balls $B_{j}$ with $(1+\delta)$-summable radii $r_{j}$ such that $r_{j} \leq 5 / k$ :

$$
A_{k}^{c} \subset \bigcup B_{j}, \quad \sum r_{j}^{1+\delta} \leq c / k .
$$

Therefore

$$
\sigma_{F}(\lambda) \leq \sum H^{2}\left(B_{j}\right)=c \sum r_{j}^{1-\delta} r_{j}^{1+\delta} \leq c k^{\delta-1} \sum r_{j}^{1+\delta} \leq c k^{\delta-2} .
$$

Expressing $k$ in terms of $\lambda$ through equation (6.4) yields the desired upper bound on $\sigma_{F}(\lambda)$ for values of $\lambda$ greater than a fixed constant and hence the conclusion of the theorem.

Remark 3. As mentioned earlier, we may combine Theorem 4.1 on uniform exceptional sets with this corollary to obtain a uniform restricted $L^{p}$ estimate for sequences of measures with uniformly bounded total mass. As a function of $\delta$, the associated exceptional sets has uniformly bounded Hausdorff premeasure of order $c \delta$. This observation yields Theorem 3 .

\section{SPACE-TIME DEFECTS}

This section concerns the size of space-time $L^{2}$ defects for approximate solution sequences for 2-D Euler. By definition these approximate solution sequences have uniformly bounded local kinetic energy and vorticity

$$
\int_{|x| \leq R}|v(x, t)|^{2} d x+T M \omega(\cdot, t) \leq K
$$

with $K$ a fixed constant for arbitrary $R, T>0$ with $0 \leq t \leq T$. Here we prove the theorem on space-time defects in approximate solution sequences for 2-D Euler stated in the Introduction. The strategy of the proof combines temporal compactness for Riesz potentials and Hölder estimates in time together with the argument for elliptic sequences at fixed time presented in the last three sections. The compactness estimates and Hölder estimates in time are valid for any approximate solution sequences for 2-D Euler and involve various interpolation arguments. These lemmas are proved in the Appendix so that the main argument is not interrupted. Next, we give the proof of Theorem 1 from the Introduction.

Given the sequence of measures, we utilize the associated Riesz potentials,

$$
\varphi(x, t)=\int|x-y|^{-\delta}|\omega|(y, t) .
$$

The following lemma generalizes the uniformization theorem, proved earlier in $\S 4$ for elliptic sequences. 
Lemma 7.1. Fix $\delta$ and $\gamma$ such that $\gamma>2 \delta$. Given a family of solutions satisfying (7.1) there exists a subsequence $u_{k}$ with the following property. For each $t$ there exists a collection of closed subsets $F_{r}(t), r>0$, of $R^{2}$ such that

$$
\begin{gathered}
H_{r}^{\gamma}\left\{F_{r}^{c}(t)\right\} \leq c, \\
\varphi_{k}(x, t) \leq c(\gamma, \delta)
\end{gathered}
$$

if $x$ lies in $F_{r}(t)$ and $k \geq 1 / r$. The constant on the right of (7.2) is universal.

Remark. The pointwise bound (7.3) implies that the associated radial distribution functions

$$
\omega_{k}(s, x, t)=\left|\omega_{k}(\cdot, t)\right|\left\{B_{s}(x)\right\}
$$

exhibit temporally uniform $\delta$-order decay if $x$ lies in the complement of the exceptional set $F_{r}^{c}(t)$.

Proof. First we apply Lemma A.2 from the Appendix, which establishes compactness of the Riesz potentials in spaces of the form $X=C\left\{[0, T] ; W^{s, p}\right\}$, $(\delta+s) p<2$. Taking $s=1$ and $p=2 /(2 \delta+1)$ for example guarantees the existence of a sequence $\varphi_{j}$ in $X$ and an element $\varphi$ on $X$ such that $\varphi_{j}$ converges to $\varphi$ in $X$. In order to produce the exceptional sets $F_{r}^{c}(t)$ for all $t$ and $r$ it suffices to select a subsequence $\varphi_{k}$ that rapidly converges to $\varphi$. For this purpose let

$$
\alpha_{j}=\sup _{0<t<T} \int\left|\nabla \varphi_{j}(x, t)-\nabla \varphi(x, t)\right|^{p} d x .
$$

As a consequence of Remark 1 in $\S 5$ it suffices to select a subsequence $\alpha_{k}$ with certain monotonicity properties such that $\alpha_{k}^{p}$ is summable in order to construct the exceptional sets. We immediately have

$$
\int\left|\nabla \varphi_{k}\left(x, t_{0}\right)-\nabla \varphi\left(x, t_{0}\right)\right|^{p} d x \leq \alpha_{k}
$$

for each $t_{0}$. Thus $\varphi(\cdot, t)$ converges fast enough to $\varphi\left(\cdot, t_{0}\right)$ in $W^{1, p}$ for each $t_{0}$.

We conclude from uniformization Theorem 4.1 that there exists an appropriate family $F_{r}^{c}\left(t_{0}\right)$ of exceptional sets for each $t_{0}$ if $k \geq 1 / r$.

With the above facts as background, we next formulate a general theorem. This theorem yields Theorem 1 for approximate solution sequences for 2-D Euler stated in the Introduction as an immediate corollary. We develop this corollary after we state Theorem 7.1 .

Theorem 7.1. Fix positive numbers $\varepsilon, \gamma, \alpha, p$, and $q$ such that $1<q<p$. Suppose $\{v\}$ is a 2-D Euler sequence which satisfies (7.1) and the following property. For every $t$ in $[0, T]$ there exists a family of closed sets $F_{r}(t), r>0$, in $R^{2}$ such that

$$
H_{r}^{\gamma}\left\{F_{r}^{c}(t)\right\} \leq c
$$


where $c$ is a fixed constant and all members $v$ of the sequence satisfy

$$
\begin{gathered}
\left|v\left(\cdot, t_{1}\right)-v\left(\cdot, t_{2}\right)\right|_{L^{q}} \leq c \Delta t^{\alpha}, \\
|v(\cdot, t)|_{L^{p}\left\{F_{r}(t)\right\}} \leq c .
\end{gathered}
$$

The conclusion is that if $s$ satisfies $q<s<p$ then there exists a family of closed sets $G_{r}, r>0$, in $R^{2} \times[0, T]$ such that

$$
H_{r}^{\gamma+1+\varepsilon}\left(G_{r}^{c}\right) \leq c_{1}
$$

and all members $v$ of the sequence satisfy

$$
|v|_{L^{s}\left(G_{r}\right)} \leq c_{1},
$$

where the constants depend on $c$ and $s$. Here ail $L^{p}$ norms are local over $|x| \leq R$.

First, we describe the fashion in which the above theorem yields Theorem 1, the result on Hausdorff dimension for the reduced defect measure for approximate solution sequences for 2-D Euler. We only need to verify the hypotheses in (7.4)-(7.6) with $p>2$ since (7.7), (7.8), and the fact that $v^{\varepsilon}$ converges strongly to $v$ in $L^{\tau}\left(B_{R}(0) \times[0, T]\right)$ for $\tau<2$ yield the required result on the reduced defect by simple interpolation. We have already verified the hypothesis in (7.4) through the argument in Lemma 7.1. To verify (7.6), we need to restrict to members of the sequence still denoted by $v_{k}$ which satisfy $k \geq 1 / r$ : the smaller the choice of the level $r$ the farther along the sequence one must proceed in order to achieve uniformity in the $L^{p}$ bound. The choice $k \geq 1 / r$ is a matter of indexing. With the remark below Lemma 7.1, we verify the uniform $L^{p}$-bound with $2<p<2+\delta / 1-\delta$ required in (7.6) by applying Theorem 6.1 on uniform velocity estimates from $\S 6$. Finally, in the Appendix, we verify that every approximate solution sequence for 2-D Euler satisfies the Hölder bound.

Remark 1. No constraints are required on the parameters $q$ and $\alpha$ which specify the degree of temporal regularity; $q$ may be arbitrarily close to one and $\alpha$ may be arbitrarily close to zero. The reason is that the bound on the size of the exceptional sets $F_{r}^{C}(t)$ is uniform in $t$. Weak temporal continuity conditions suffice to construct a space-time exceptional set out of a family of spatial exceptional sets.

Finally, we give the proof of Theorem 7.1.

Proof. The objective is to estimate restricted distribution functions of the form

$$
\sigma(\lambda)=m_{3}\left\{(x, t) \varepsilon G_{r} \cap[0, T]:|v| \geq \lambda\right\}
$$

where $m_{3}$ denotes 3-dimensional Lebesgue measure and $G_{r}(t)$ denotes the cross section of $G_{r}$ at time $t$.

We shall show that $\sigma \leq c \lambda^{-p}$ by estimating a Riemann sum over a uniform finite mesh $\left\{t_{j}: 1 \leq j \leq m 2^{N}\right\}$ where $m$ and $N$ are integers chosen in an 
appropriate fashion:

$$
\sigma(\lambda)=\int_{0}^{T} f d t \leq \sum_{j} \sup \left\{|f(t)|: t \in I_{j}\right\} \Delta t
$$

where

$$
\begin{aligned}
& f(t, \lambda)=m_{2}\left\{x \in G_{r}(t):|v(x, t)| \geq \lambda\right\}, \\
& I_{j}=\left\{t:\left|t-t_{j}\right| \leq \Delta t\right\} .
\end{aligned}
$$

The mesh consists of $m 2^{N}$ points $t_{j}$ that partition $[0, T]$ into intervals of equal length $\Delta t$. Thus, the $p$ th order Marcinkiewicz norm of the restriction of $u$ to $G_{r}$ is bounded by a fixed constant. The same can be said for the $L^{s}$ norm if $s<p$. Here and in the following we use the symbol $v$ to denote an arbitrary member of the sequence $v_{k}$.

The exceptional set $G_{r}$ is constructed in the following way. Fix $r$ and select a point $t_{j}$ of the mesh. Consider the spatial exceptional set $F_{j}^{c}=F_{r}^{c}\left(t_{j}\right)$. Notice that the $\gamma$-order premeasure of $F_{r}^{c}$ is bounded uniformly with respect to $r$ by (7.4). Without loss of generality we may assume that $F_{j}^{c}$ is a countable union of balls $B_{j, k}$ in $R^{2}$ with radii $r_{j, k}$ satisfying

$$
\sum r_{j, k}^{\gamma} \leq c \text { and } r_{j, k} \leq r .
$$

Associate with each ball a cylinder with height $h_{j}$ :

$$
C_{j, k}=B_{j, k} \times\left\{t:\left|t-t_{j}\right| \leq h_{j} / 2\right\} .
$$

We define the space-time exceptional set $G_{r}^{c}$ to be a union of cylinders, namely

$$
G_{r}^{c}=\bigcup_{j, k} C_{j, k}
$$

and require that the heights satisfy the following inequalities

$$
\sum h_{j}^{1+\varepsilon} \leq c \text { and } h_{j} \leq r
$$

where $c$ is a fixed constant. We first observe that the cylindrical premeasure of $G_{r}^{c}$ is bounded uniformly with respect to $r$ by virtue of (7.11) and (7.12). In general the $(\tau, \pi)$-order cylindrical Hausdorff premeasure of a set $E$ is determined by the most efficient countable cover with cylinders of height $h_{j}$ and sectional radius $r_{j}$ :

$$
H_{r}^{\tau, \pi}(E)=\inf \left\{\sum r_{j}^{\tau} h_{j}^{\pi}: E \subset \bigcup C\left(r_{j}, h_{j}\right)\right\}
$$

where $r_{j} \leq r$ and $h_{j} \leq r$. For the special case at hand we have

$$
H_{r}^{\gamma, 1+\varepsilon}\left(G_{r}^{c}\right) \leq \sum \sum r_{j, k}^{\gamma} h_{j}^{1+\varepsilon} \leq c \sum h_{j}^{1+\varepsilon} \leq c
$$

using (7.11) and (7.12). The definitions of $h_{j}$ and $t_{j}$ are given below.

Cylindrical and spherical premeasure satisfy the following inequality

$$
H_{r}^{\tau+\pi}(E) \leq c H_{r}^{\tau, \pi}(E)
$$


where the constant depends only on $\tau, \pi$ and the dimension of the space. Thus, the upper bound (7.4) on the $\gamma+1+\varepsilon$-order premeasure of $G_{r}^{c}$ follows from (7.13) and (7.14).

In order to obtain the desired upper bound on $\sigma$ we shall first show that

$$
\sigma(\lambda) \leq c T \lambda^{-p}+c T \Delta t^{\alpha q} \lambda^{-q} .
$$

To this end, we shall show that

$$
f(t, \lambda) \leq A_{j}+B_{j}
$$

if $t \in I_{j}$ where

$$
A_{j}=c \lambda^{-p} \quad \text { and } \quad B_{j}=c\left|t-t_{j}\right|^{d q} \lambda^{-q} .
$$

Substitution of the pointwise bounds (7.17) into the Riemann sum yields (7.15).

In order to prove (7.16) we first observe that if $t$ lies in $I_{j}$ then the $t$-section of $G_{r}^{c}$ contains $F_{j}^{c}$. Thus

$$
f(t, \lambda) \leq m_{2}\left\{x \in F_{j}:|v(x, t)| \geq \lambda\right\}
$$

if $t \in I_{j}$. The triangle inequality implies that

$$
f(t, \lambda) \leq m_{2}\left\{x \in F_{j}:\left|v\left(x, t_{j}\right)\right|>\lambda / 2\right\}+m_{2}\left\{x \in F_{j}:\left|v(x, t)-v\left(x, t_{j}\right)\right|>\lambda / 2\right\}
$$

and therefore (7.16) holds with

$$
\begin{aligned}
& A_{j}=m_{2}\left\{x \in F_{j}:\left|v\left(x, t_{j}\right)\right|>\lambda / 2\right\}, \\
& B_{j}=m_{2}\left\{x \in R^{2}:\left|v(x, t)-v\left(x, t_{j}\right)\right|>\lambda / 2\right\}
\end{aligned}
$$

if $t \in I_{j}$. The restricted $L^{p}$ bound (7.9) yields the desired bound on the spatial distribution function of the restriction of $v$ to $F_{j}$ and hence on $A_{j}$. The temporal Hölder estimate (7.5) yields the desired bound on $B_{j}$. Thus (7.17) holds.

Finally, the integer $N$ is chosen so that the factor $\Delta t^{\alpha q}$ in (7.15) compensates for the large term $\lambda^{-q}$ on the right side of (7.15). We rewrite (7.15) in the form

$$
\sigma \leq c T \lambda^{-p}+c T \lambda^{-p} \beta
$$

where

$$
\beta=\Delta t^{\alpha q} \lambda^{p-q}=\left(2^{-N-1} T\right)^{\alpha q} \lambda^{p-q} .
$$

We fix $\lambda$ and choose $N$ sufficiently large to guarantee that $\beta$ is dominated by a fixed constant.

It is convenient to regard the set of points $t_{j}$ as a union of a sequence of finite meshes $M_{k}$ defined inductively as follows. Assume with loss of generality that $T / r$ is an integer and let $m=T / r$. Take $M_{1}$ to consist of $t_{j}=j r$ where $1 \leq j \leq m$. Given $M_{k}$ define $M_{k+1}=M_{k} \cup C_{k}$ where $C_{k}$ denotes the collection of midpoints of the subintervals of $(0, T)$ determined by $M_{k}$. Notice that $M_{k+1}$ contains twice as many points as $M_{k}$. Thus, $M_{k}$ consists of $m 2^{k}$ points $t_{j}, 1 \leq j \leq m 2^{k}$, which partition $(0, T)$ onto intervals of 
equal length and may be regarded as a disjoint union, $M_{k}=\bigcup_{i=0}^{k-1} C_{i}$ by taking $C_{0}=M_{1}$.

If $t_{j}$ lies in $C_{i}$, define the corresponding $h_{j}$ to be $r / 2^{i}$, i.e., the distance between consecutive points of $C_{i}$. Thus the sum of the heights associated with points of $C_{i}$ equals $T$ and

$$
\begin{aligned}
\sum h_{l}^{1+\varepsilon} & =\sum_{i} \sum\left\{h_{j}^{1+\varepsilon}: t_{j} \in C_{i}\right\} \leq \sum_{i}\left(r 2^{-i}\right) \sum_{j}\left\{h_{j}: t_{j} \varepsilon C_{i}\right\} \\
& \leq T \sum_{i}\left(r 2^{-i}\right)^{\varepsilon} \leq \mathrm{const} .
\end{aligned}
$$

\section{APPENDIX: TRUNCATION, INTERPOLATION,} AND HÖLDER CONTINUITY IN TIME

Here we verify that we can truncate approximate solution sequences for 2-D Euler which satisfy the conditions from Definition 1.1 of [4]. We also prove the temporal Hölder continuity estimate in (7.5) needed in the proof of Theorem 1 as well as the temporal compactness lemma for Riesz potentials which we used to begin the proof of Lemma 7.1.

The requirement that the velocity field vanishes uniformly at infinity for the approximate sequence of velocity fields guarantees that the stream function $\psi^{\varepsilon}$ satisfying

$$
\Delta \psi^{\varepsilon}=\omega^{\varepsilon}(x, t), \quad v^{\varepsilon}=\nabla^{\perp} \psi^{\varepsilon}
$$

splits into a sum of stream functions

$$
\psi^{\varepsilon}=\bar{\psi}^{\varepsilon}(|x|, t)+\widetilde{\psi}^{\varepsilon}(x, t)
$$

with

$$
\sup _{|x| \leq R} \sum_{|\alpha| \leq 2}\left|D^{\alpha} \bar{\psi}^{\varepsilon}(|x|, t)\right| \leq c
$$

and

$$
\tilde{\psi}^{\varepsilon}(x, t)=\frac{1}{2 \pi} \int \log |x-y| \widetilde{\omega}^{\varepsilon}(y, t) d y .
$$

Here $\tilde{\omega}^{\varepsilon}(y, t)$ is a smooth rapidly decreasing function with

$$
\int \widetilde{\omega}^{\varepsilon}(y, t) d y=0, \quad \int\left|\widetilde{\omega}^{\varepsilon}\right| d y \leq c .
$$

For a fixed function $\rho$ with $\rho \in C_{0}^{\infty}, \rho \geq 0, \operatorname{supp} \rho \subseteq\{x|| x \mid<R+I\}, \rho \equiv I$ for $|x|<R$, we consider $\rho \psi^{\varepsilon}$ which satisfies

$$
\Delta\left(\rho \psi^{\varepsilon}\right)=\rho \omega^{\varepsilon}+2 \nabla \rho \cdot \nabla \psi^{\varepsilon}+\Delta \rho \psi^{\varepsilon}
$$

and

$$
\nabla^{\perp}\left(\rho \psi^{\varepsilon}\right)=\rho v^{\varepsilon}+\psi^{\varepsilon} \nabla^{\perp} \rho .
$$


We need to verify a uniform $L^{2}$ bound as in (1.2) for $\nabla^{\perp}\left(\rho \psi^{\varepsilon}\right)$ and a uniform $L^{1}$ bound for $\Delta\left(\rho \psi^{\varepsilon}\right)$. The stream functions $\psi^{\varepsilon}$ are only determined within an additive constant so we modify $\widetilde{\psi}^{\varepsilon}(x, t)$ by a constant as follows

$$
\begin{aligned}
2 \pi \tilde{\psi}^{\varepsilon}= & \left\{\int_{|x-y| \leq 4 R+4} \log |x-y| \widetilde{\omega}^{\varepsilon}(y, t) d y\right\} \\
& +\left\{\int_{|x-y| \geq 4 R+4} \log |x-y| \widetilde{\omega}^{\varepsilon}(y, t) d y-\int \log ^{+}|y| \tilde{\omega}^{\varepsilon}(y, t) d y\right\} \\
= & \widetilde{\psi}_{1}^{\varepsilon}+\widetilde{\psi}_{2}^{\varepsilon} .
\end{aligned}
$$

The first term in brackets, $\widetilde{\psi}_{1}^{\varepsilon}$, is a convolution and satisfies

$$
\left\|\tilde{\psi}_{1}^{\varepsilon}\right\|_{L^{q^{\prime}}} \leq c_{q^{\prime}}\left\|\tilde{\omega}^{\varepsilon}\right\|_{L^{1}} \leq c_{q}
$$

for any $q^{\prime}>1$ while the second term in brackets satisfies

$$
\max _{|x| \leq R} \sum_{|\alpha| \leq 1}\left|D^{\alpha} \widetilde{\psi}_{2}^{\varepsilon}\right| \leq C(R)\left\|\tilde{\omega}^{\varepsilon}\right\|_{L^{1}} \leq C(R) .
$$

The required uniform $L^{1}$ bound in (A-2) and the uniform $L^{2}$ in (A-3) follow directly from the estimates in (A-4), (A-5), and the corresponding bounds in (1.2) and (1.3) on $v^{\varepsilon}$ and $\omega^{\varepsilon}$.

Next we prove the Hölder continuity estimate from (7.5).

Lemma A.1. Let $v^{\varepsilon}$ be an approximate solution sequence of velocity fields for 2-D Euler, then for any $p$ with $1 \leq p<2$, there is a number $\alpha, 0<\alpha<1$, so that

$$
\left\|v^{\varepsilon}\left(t_{1}\right)-v^{\varepsilon}\left(t_{2}\right)\right\|_{L^{p}\left(B_{R}\right)} \leq C\left|t_{1}-t_{2}\right|^{\alpha}
$$

Proof. From the definition of approximate solution sequence (see Definition 1.1 of [4]), $\rho v^{\varepsilon}$ is a uniformly Lipschitz function with values in $W_{\text {loc }}^{-L, 2}\left(R^{2}\right) \subseteq$ $W_{\text {loc }}^{-L, p}\left(R^{2}\right)$ with $p \leq 2$ for some number $L>0$ and any $\rho \in C_{0}^{\infty}\left(R^{2}\right)$. This guarantees that for $p \leq 2$,

$$
\left\|\rho v^{\varepsilon}\left(t_{1}\right)-\rho v^{\varepsilon}\left(t_{2}\right)\right\|_{-L, p} \leq C\left|t_{1}-t_{2}\right|
$$

for some fixed constant $C$. The function $\rho v^{\varepsilon}$ satisfies the elliptic system

$$
\begin{aligned}
\operatorname{curl}\left(\rho v^{\varepsilon}\right) & =\nabla^{\perp} \rho \cdot v^{\varepsilon}+\rho \omega^{\varepsilon} \equiv F_{1}^{\varepsilon}, \\
\operatorname{div}\left(\rho v^{\varepsilon}\right) & =\nabla \rho \cdot v^{\varepsilon}=F_{2}^{\varepsilon} .
\end{aligned}
$$

The inhomogeneous term of the right-hand side of (A-8) are uniformly bounded in $L^{1}$ as a consequence of (1.2) and (1.3). By Sobolev's lemma, $W^{s, p^{\prime}} \rightarrow C$ for $s>N / p^{\prime}$, so by duality, $L^{1} \rightarrow W^{-s, p}$ for $s>N((p-1) / p), p>1$, and therefore

$$
\left\|F^{\varepsilon}\right\|_{-s, p} \leq C \quad \text { for } s>2((p-1) / p)
$$


and $p>I$. Solutions of the elliptic system in (A-8) gain a derivative, therefore

$$
\left\|\rho v^{\varepsilon}\right\|_{1-s, p} \leq C\left(\left\|F^{\varepsilon}\right\|_{-s-p}+\left\|\rho v^{\varepsilon}\right\|_{L^{2}}\right) \leq C
$$

for $s>2((p-1) / p)$. Thus, for $1<p<2$, we have for some $s_{1}=1-s>0$,

$$
\left\|\rho v^{\varepsilon}(t)\right\|_{s_{1}, p} \leq C, \quad 0 \leq t \leq T .
$$

From the interpolation inequality to be discussed below, there is a number $\alpha$ with $0<\alpha<1$ so that

$$
(\mathrm{A}-11)\left\|\rho v^{\varepsilon}\left(t_{1}\right)-\rho v^{\varepsilon}\left(t_{2}\right)\right\|_{L^{p}} \leq C\left\|\rho v^{\varepsilon}\left(t_{1}\right)-\rho v^{\varepsilon}\left(t_{2}\right)\right\|_{S_{1}, p}^{1-\alpha}\left\|\rho v^{\varepsilon}\left(t_{1}\right)-\rho v^{\varepsilon}\left(t_{2}\right)\right\|_{-L, p}^{\alpha} .
$$

With the Lipschitz bound in (A-7) and the uniform bound in (A-10), we verify the Hölder estimate in (A-6) through the interpolation inequality in (A-11).

In Lemma 7.1, we always work with a truncated vorticity with support contained inside a ball $B_{R}=\{x|| x \mid<R\}$. The Riesz potentials are used for bounded values of $|x|$ in order to apply the uniformization theorem. It is well known that Bessel potentials are equivalent to Riesz potentials of the same order for bounded values of $x$ when applied to positive measures with support inside a fixed bounded set (see the Appendix of [1]). Thus, we prove a temporal compactness lemma for the corresponding Bessel potentials,

$$
\varphi=\int G_{\delta}(x-y) d|\omega|(y), \quad \delta>0
$$

with the kernel $G_{\delta}$ given explicitly on page 132 of [8] and with the Fourier transform of $G_{\delta}, \hat{G}_{\delta}=\left(1+|\xi|^{2}\right)^{-\delta / 2}$. We have the following compactness lemma for Bessel potentials.

Lemma A.2. Fix positive numbers $\delta$, $s$, and $p$ such that $p>1$ and $(\delta+s) p<$ 2 , if $v^{\varepsilon}$ is a sequence of approximate velocity fields for 2-D Euler and $\omega^{\varepsilon}$ is the corresponding truncated vorticity field associated with $\rho v^{\varepsilon}$ with $\rho \in C_{0}^{\infty}\left(R^{2}\right)$, then there exists a subsequence $v^{\varepsilon}$ for which the associated Bessel potentials

$$
\varphi^{\varepsilon}(x, t)=\int G_{\delta}(x-y) d\left|\omega^{\varepsilon}\right|(y)
$$

converge to a function $\varphi$ in $C\left([0, T], W_{\mathrm{loc}}^{s, p}\right)$, i.e.,

$$
\lim _{\varepsilon \rightarrow 0} \sup _{0 \leq t \leq T}\left\|\rho_{1} \varphi^{\varepsilon}(x, t)-\rho_{1} \varphi(x, t)\right\|_{s, p}=0
$$

for every $\rho_{1} \in C_{0}^{\infty}$.

In $\S 4$, we already verified that the Bessel potentials of uniformly bounded measures with mass in $B_{R}$ lie in a bounded subset of $W_{\text {loc }}^{\tau, p}\left(R^{N}\right)$ for any $\tau$ with 
$(\delta+\tau) p<N$. Thus, $\rho_{1} \varphi$ lies in a bounded subset of $W_{\text {loc }}^{\tau, p}$. Since the injection, $i: W_{\text {loc }}^{\tau, p} \rightarrow W_{\text {loc }}^{s, p}$ is a compact mapping for $\tau>s$ and $(\delta+\tau) p<N,(\delta+s) p<$ $N$, by the Lions-Aubin lemma, we only need to verify the temporal Hölder continuity of $\rho_{1} \varphi$ in some negative normal space, $W^{-R, p}$. From Lemma A.1, the truncated vorticity $\omega^{\varepsilon}$ satisfies the temporal Hölder estimate

$$
\left\|\omega^{\varepsilon}\left(t_{1}\right)-\omega^{\varepsilon}\left(t_{2}\right)\right\|_{-1, p} \leq c\left|t_{1}-t_{2}\right|^{\alpha} .
$$

It is well known [1, Chapter VII] that the Bessel potential $G_{\delta}$ maps $W^{-1, p}$ boundedly into $W^{-1+\delta-\varepsilon, p}$ for any $\varepsilon>0$; this fact, coupled with the estimate in (A-13), yields the negative-norm temporal Hölder estimate required to complete the proof of Lemma A.2.

It remains to discuss the interpolation inequality in (A-11). We utilize the spaces $L^{s, p}, 1<p<\infty$, defined through Bessel potentials as the tempered distributions $u$ so that the inverse Fourier transform of $\left(1+|\xi|^{2}\right)^{s / 2} \hat{u}(\xi)$ belongs to $L^{p}$ with norm $\|u\|_{L^{s, p}}$ (see Stein [8] and Adams [1] for the facts we quote below). The spaces satisfy the estimates

$$
\begin{aligned}
& \|u\|_{L^{s_{2}, p}} \leq\|u\|_{L^{s_{1}, p}}, \quad s_{2} \leq s_{1}, \\
& \|u\|_{s, p} \leq C_{\varepsilon}\|u\|_{L^{s+\varepsilon, p}}, \\
& \|u\|_{L^{s-\varepsilon, p}} \leq C_{\varepsilon}\|u\|_{s, p} \quad \text { any } \varepsilon>0 .
\end{aligned}
$$

The constants in the comparison between $W^{s, p}$ and $L^{S, p}$ blow up in general as $\varepsilon \rightarrow 0$. The $L^{s, p}$ spaces satisfy the interpolation inequality

$$
\|u\|_{L^{s, p}} \leq C\|u\|_{L^{s_{1}, p}}^{1-\theta}\|u\|_{L^{s_{2}, p}}^{\theta}
$$

for $s_{2}<s<s_{1}$ and appropriate $\theta$ with $0<\theta<1$. The facts in (A-14) and (A-15) combine to yield (A-11). The proof of (A-15) proceeds in a standard fashion. We pick $\varphi \in C_{0}^{\infty}$ with $\varphi(\xi) \equiv 1$ for $|\xi|<1$ and $\operatorname{supp} \varphi \subseteq\{\xi|| \xi \mid>2\}$. We write for $0<\varepsilon \leq 1$,

$$
\begin{aligned}
\left(1+|\xi|^{2}\right)^{s} \hat{u}(\xi)= & \left\{\left(1+|\xi|^{2}\right)^{\left(s-s_{1}\right) / 2}\left(1-\varphi(\varepsilon \xi)\left(1+|\xi|^{2}\right)^{s_{1} / 2} \hat{u}(\xi)\right\}\right. \\
& +\left\{\left(1+|\xi|^{2}\right)^{\left(s-s_{2}\right) / 2} \varphi(\varepsilon \xi)\left(1+|\xi|^{2}\right)^{s_{2} / 2} \hat{u}(\xi)\right\}
\end{aligned}
$$

From the explicit $L^{p}$ bounds guaranteed by the Marcinkiewicz multiplier theorem (see [8]) and (A-16), we deduce the bound

$$
\|u\|_{L^{s, p}} \leq C\left(\varepsilon^{s_{1}-s}\|u\|_{L^{s_{1}, p}}+\varepsilon^{s_{2}-s}\|u\|_{L^{s_{2}, p}}\right) .
$$

By minimizing the right-hand side over $\varepsilon$ with $0<\varepsilon \leq 1$, we obtain (A-15).

\section{REFERENCES}

1. R. Adams, Sobolev spaces, Academic Press, New York, 1975.

2. R. Adams and N. Meyers, Bessel potentials. Inclusion relations among classes of exceptional sets, Indiana Math. J. 22 (1973), 873-905.

3. R. DiPerna and A. Majda, Oscillations and concentrations in weak solutions of the incompressible fluid equations, Commun. Math. Phys. 108 (1987), 667-689. 
4. $\ldots$, Concentrations in regularizations for 2-D incompressible flow, Comm. Pure Appl. Math. 60 (1987), 301-345.

5. H. Federer and W. P. Ziemer, The Lebesgue set of a function whose distributional derivatives are pth-power summable, Indiana Univ. Math. J. 22 (1972), 139-158.

6. P. L. Lions, The concentration-compactness principle in the calculus of variations: the locally compact case, Parts I and II, Ann. Inst. H. Poincaré, 1984, 109-145 and 223-283.

7. Parts I and II, Riv. Mat. Iberoamericana I (1984), 145-201 and I (1985), 45-121.

8. E. M. Stein, Singular integrals and differentiability properties of functions, Princeton University Press, N.J., 1970.

Department of Mathematics, University of California, Berkeley, California 94720

Department of Mathematics and Program in Applied and Computational Mathematics, Princeton University, Princeton, New Jersey 08544 